\title{
CHORDAL CUBIC SYSTEMS
}

\author{
MARC CARBONELl AND JAUME LLIBRE
}

\begin{abstract}
We classify the phase portraits of the cubic systems in the plane such that they do not have finite critical points, and the critical points on the equator of the Poincare sphere are isolated and have linear part nonidentically zero.
\end{abstract}

\section{Introduction}

We consider cubic systems (CS, for abbreviation), i.e. two-dimensional autonomous systems of differential equations of the form

$$
\dot{x}=P(x, y) \quad, \quad \dot{y}=Q(x, y),
$$

where $P$ and $Q$ are real polynomials such that $\max \{$ degree $P$, degree $Q\}=3$. If a CS has no finite critical points, then it will be called chordal cubic system. We shall denote by CCS the chordal cubic systems such that they only have isolated critical points on the equator of the Poincaré sphere (see [8], [16] or Appendix $A$ of [6]) and the linear part of these critical points are not identically zero. The chordal systems were studied by Kaplan [10], [11]. The name of chordal system is due to the fact that a such system has all its solutions starting and endding at the equator of the Poincare sphere.

In this paper we give a classification of the phase portraits (on the Poincaré disk) of CCS. A complete study for the chordal quadratic systems has been done by Gasull, Sheng Li-Ren and Llibre in [7].

Our main result is the following one.

Theorem. The phase portrait of a CCS is homeomorphic (except, perhaps the orientation) to one of the separatrix configurations shown in Figure 1. Furthermore, all the separatrix configurations of Figure 1 are realizable for chordal cubic systems. 

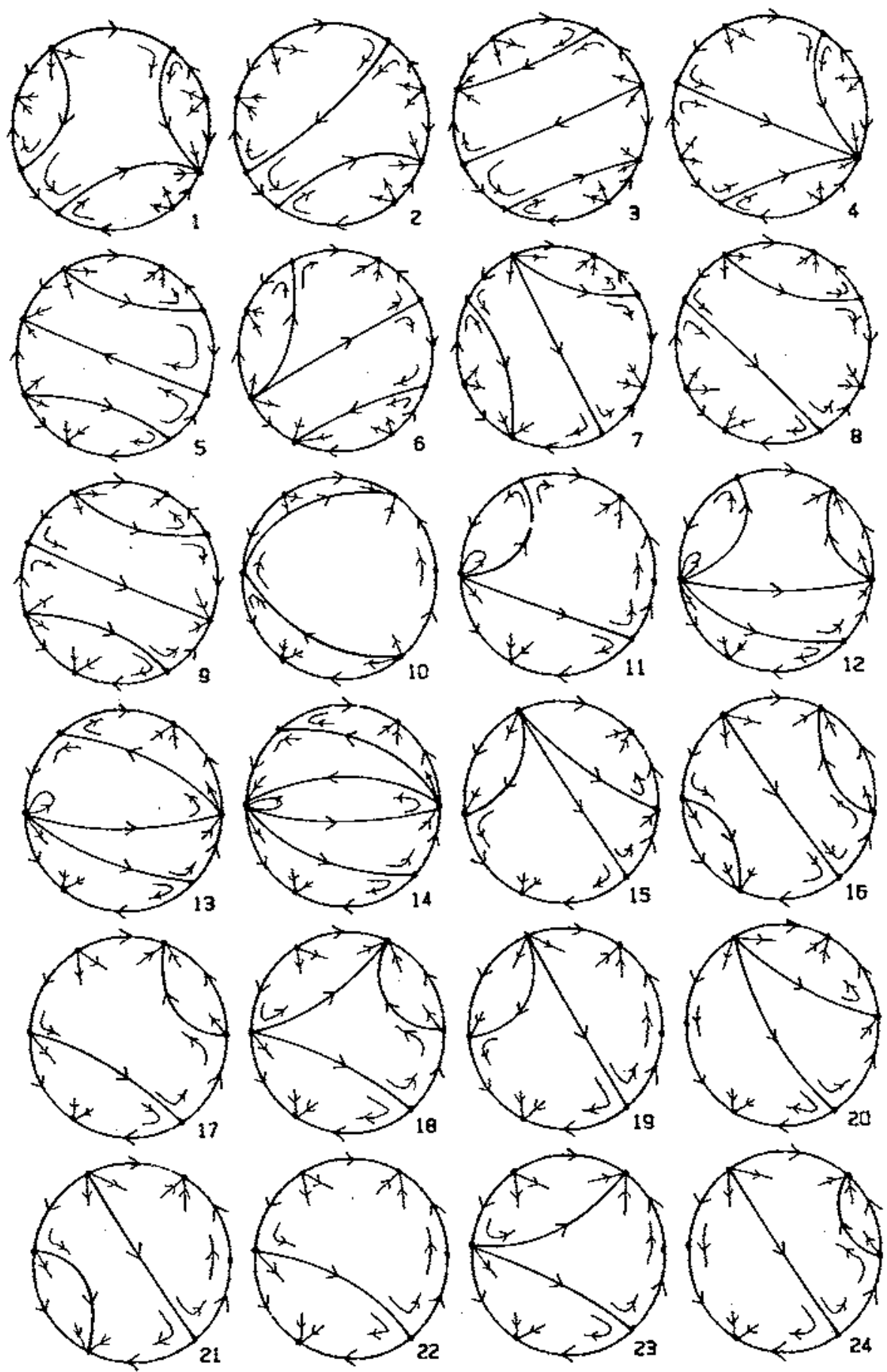

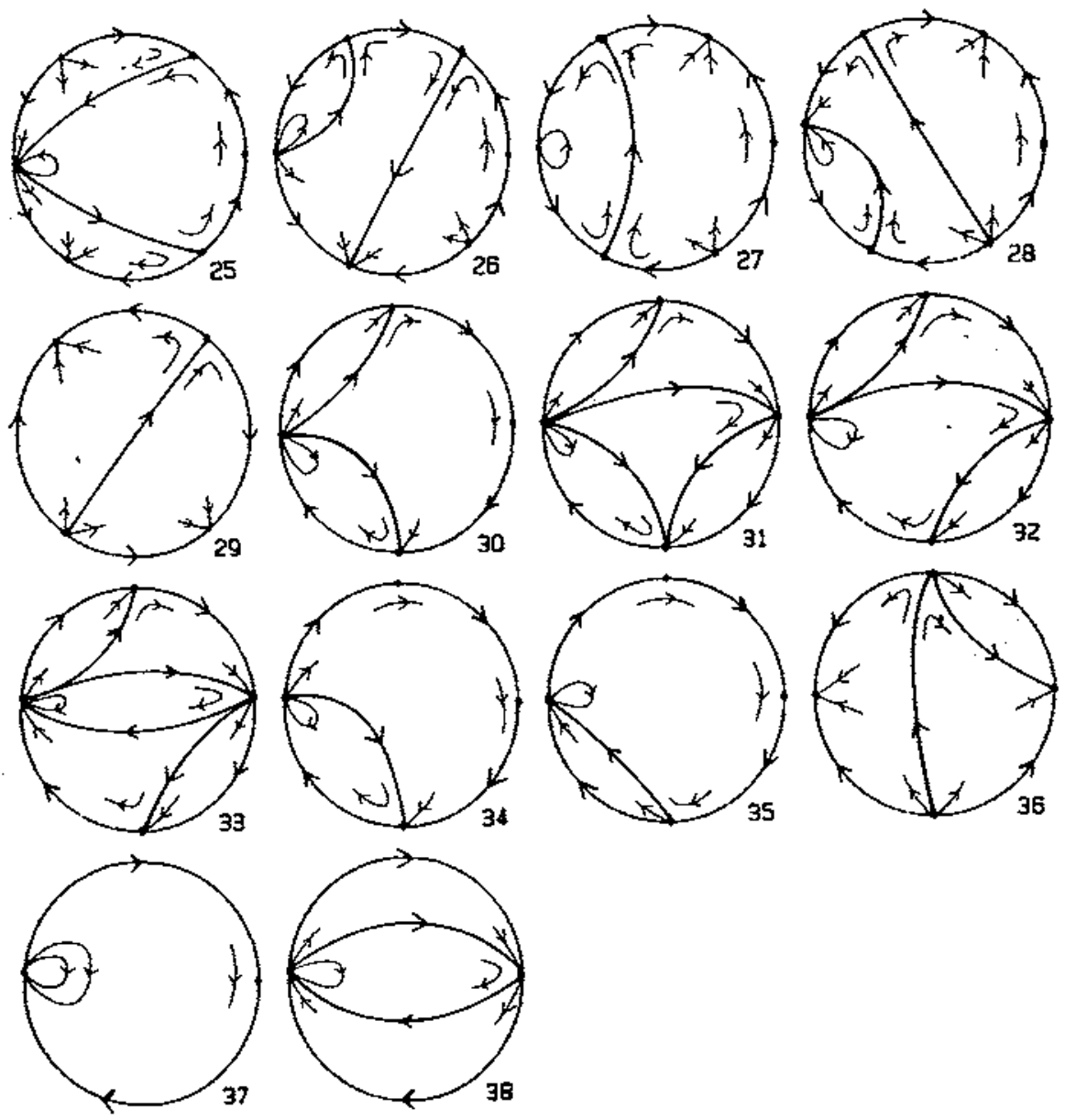

Figure 1. The phase portraits of a CCS (except, perhaps the orientation).

In what follows, when we refer to the phase portrait $i$ of Figure $1,1 \leq i \leq 38$, we shall only say the phose portrait $i$.

We note that the realizations of phase portraits $12,13,14,31,32$ and 33 uses infinite critical points with linear part identically zero.

A non singular differential equation in two real variables defines a foliation of the plane. It is well known that the topological classification of such foliations depends only of the number of inseparable leaves and the way they are 
distributed in the plane (see [10] or [9]). Two leaves (or trajectories) $L_{1}$ and $L_{2}$ are said to be inseparable if for any arcs $T_{1}$ and $T_{2}$ respectively transversal to $L_{1}$ and $L_{2}$ there are leaves which intersect both $T_{1}$ and $T_{2}$ (see Figure 2).

For polynomial foliations of degree $n$, i.e. defined by a polynomial vector field $(P, Q)$, where $\max \{$ degree $P$, degree $Q\}=n$, it is known that the number of inseparable leaves is at most $2 n$ (see [13] or [15]). Actually, a construction leading to examples with $2 n-4$ inseparable leaves for all $n \geq 4$ can be already found in [14]. In [3] it is claimed that the case $n=2$ has at most 2 inseparable leaves. This claim is not true because in [7] the quadratic system $\dot{x}=1+x y$, $\dot{y}=m^{-1} y^{2}$, with $m<-1$ has 3 inseparable leaves: $y=0$ and the two branches of the hyperbola $x y=-m /(m+1)$. Also from [7] it follows that 3 is the maximum number of inseparable leaves when $n=2$. In [5] it is proved that 3 is again the maximum number of inseparable leaves when $n=3$.

Note that our chordal cubic systems provide examples of polynomial foliations of degree 3 in the plane with 3 inseparable leaves, see for instance phase portrait 5 .

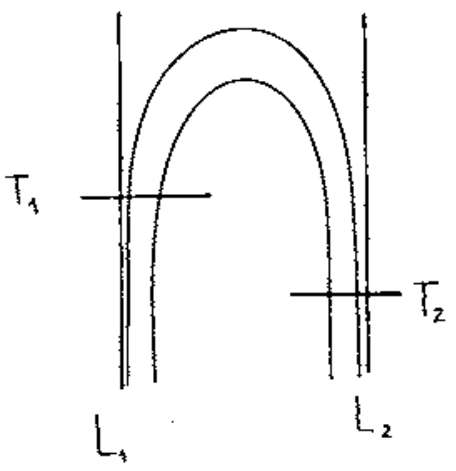

Figure 2.

\section{Classification of cubic systems}

In this section we will state the main results on the classification of CS due to Cima and Llibre [3].

Definition. A binary quartic form $f(x, y)$ is a real homogeneous polynomial of degree 4 in the variables $x$ and $y$.

Theorem 2.1. Any binary quartic form by means of a linear change of variables, can be written as one of the ten binary quartic forms contained in Table 1 . 
Let $g(x, y)$ be the binary quartic form $x Q_{3}(x, y)-y P_{3}(x, y)$ associated to cubic system (1.1), where $P_{3}$ and $Q_{3}$ are the homogeneous polynomials of degree 3 of $P$ and $Q$, respectively. From Appendix A of [6] the zeros of $g(x, y)$ give us the infinite critical points of this CS. Then from Theorem 2.1 we obtain the next result.

Corollary 2.2. By using a linear change of variables, any binary quartic form $g$ associated to a CS is one of the binary quartic forms of Table 1 .

From the correspondance between cubic systems and binary quartic forms, and from Theorem 2.1 we have the following result.

Theorem 2.3. Any CS, by means of a linear change of variables, can be written as one of the ten CS contained in Table 2.

In what follows we will denote by $\operatorname{CCS}(J)$ to any CCS of type $(J)$ of Theorem 2.3 , where $J \in\{I, I I, \ldots, X\}$.

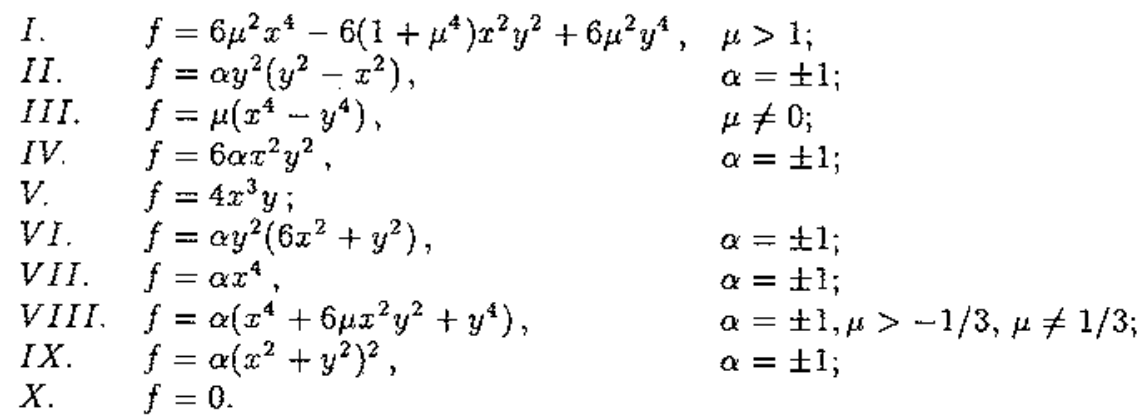

Table 1. Classification of the binary quartic forms.

(I)

$$
\begin{aligned}
& \dot{x}=q_{1}-3\left(1+\mu^{4}\right) x^{2} y+6 \mu^{2} y^{3}+x p, \\
& \dot{y}=q_{2}-6 \mu^{2} x^{3}+3\left(1+\mu^{4}\right) x y^{2}+y p, \\
& \mu>1 ;
\end{aligned}
$$

$$
\begin{aligned}
& \dot{x}=q_{1}+\alpha\left[(-1 / 2) x^{2} y+y^{3}\right]+x p, \\
& \dot{y}=q_{2}+\alpha(1 / 2) x y^{2}+y p \\
& \alpha= \pm 1
\end{aligned}
$$




$$
\begin{aligned}
& \dot{x}=q_{1}-\mu y^{3}+x p, \\
& \dot{y}=q_{2}-\mu x^{3}+y p, \\
& \mu \neq 0
\end{aligned}
$$

$$
\begin{aligned}
& \dot{x}=q_{1}+3 \alpha x^{2} y+x p \\
& \dot{y}=q_{2}-3 \alpha x y^{2}+y p \\
& \alpha= \pm 1
\end{aligned}
$$

$$
\begin{aligned}
& \dot{x}=q_{1}+2 x^{3}+x p, \\
& \dot{y}=q_{2}-2 x^{2} y+y p,
\end{aligned}
$$

$$
\begin{aligned}
& \dot{x}=q_{1}+\alpha\left(3 x^{2} y+y^{3}\right)+x p \\
& \dot{y}=q_{2}-3 \alpha x y^{2}+y p \\
& \alpha= \pm 1
\end{aligned}
$$

$$
\begin{aligned}
& \dot{x}=q_{1}+x p, \\
& \dot{y}=q_{2}-\alpha x^{3}+y p, \\
& \alpha= \pm 1 ;
\end{aligned}
$$

(VIII)

$$
\begin{aligned}
& \dot{x}=q_{1}+\alpha\left(3 \mu x^{2} y+y^{3}\right)+x p \\
& \dot{y}=q_{2}-\alpha\left(x^{3}+3 \mu x y^{2}\right)+y p \\
& \alpha= \pm 1, \mu>-1 / 3, \mu \neq 1 / 3
\end{aligned}
$$

$$
\begin{aligned}
& \dot{x}=q_{1}+\alpha\left(x^{2} y+y^{3}\right)+x p \\
& \dot{y}=q_{2}-\alpha\left(x^{3}+x^{2} y\right)+y p \\
& \alpha= \pm 1
\end{aligned}
$$

$$
\begin{aligned}
& \dot{x}=q_{1}+x p, \\
& \dot{y}=q_{2}+y p ;
\end{aligned}
$$

where

$$
\begin{aligned}
& p=p_{1} x^{2}+p_{2} x y+p_{3} y^{2}, \text { and } \\
& q_{k}=d_{k}+a_{k} x+b_{k} y+l_{k} x^{2}+m_{k} x y+n_{k} y^{2}, \text { for } k=1,2
\end{aligned}
$$

Table 2. Classification of the cubic systems. 


\section{Critical points}

To obtain the possible phase portraits of chordal cubic systems we need to know the local behaviour of their infinite critical points. To study this infinite critical points, we use the following classification: If $p$ is an isolated critical point of a vector field $X$, we say that $p$ is of type

$E$ if det $D X(p) \neq 0$, (non-degenerate and elementary);

$D E$ if $\operatorname{det} D X(p)=0$ and $\operatorname{tr} D X(p) \neq 0$, (degenerate and elementary);

$\mathrm{NE}$ if $\operatorname{det} D X(p)=\operatorname{tr} D X(p)=0$ and $D X(p) \not \equiv 0$, (non-elementary);

$\mathrm{Z}$ if $D X(p) \equiv 0$,

where $D X(p)$ denotes the linear part of $X$ at $p$.

Thus to study the infinite critical points of a CCS we shall use the Theorems E, DE, NE and the Poincaré-Hopf theorem (see Appendix).

\subsection{Degenerate and elementary critical points.}

This subsection deals with the local behaviour of the origin of systems $U_{1}$ and $U_{2}$ (see Appendix $A$ of $[6]$ ) when it is a degenerate and elementary critical point (DE).

From now on we shall denote by $(y, z)$ the coordinates $\left(z_{1}, z_{2}\right)=F_{1}\left(y_{1}, y_{2}, y_{3}\right)$ where $\left(y_{1}, y_{2}, y_{3}\right) \in U_{1}$ and by $(x, z)$ the coordinates $\left(z_{1}, z_{2}\right)=F_{2}\left(y_{1}, y_{2}, y_{3}\right)$ where $\left(y_{1}, y_{2}, y_{3}\right) \in U_{2}$.

Proposition 3.1.1. Assume that $p_{1} \neq 0$. Then the origin of system $U_{1}$ associated to systems (II), (IV) and (VI) is a saddle-node of type DE. Furthermore, its local behaviour is shown in Figure $\&$ for system (II) and in Figure 4 for systems (IV) and (VI).

Proof: The expression of system $U_{1}$ associated to systems (II), (IV) and (VI) is the following one,

$$
\begin{aligned}
& \dot{y}=l_{2} z+Y(y, z), \\
& \dot{z}=-p_{1} z+Z(y, z),
\end{aligned}
$$

where $Y$ and $Z$ are polynomials in $y$ and $z$ of degree at least two. It is clear that if $p_{1} \neq 0$ then the origin of system (3.1.1) is a critical point of type DE. To analyse its local behaviour we shall use the Theorem DE (see Appendix). First, we need to consider the change of variables, $y_{1}=y+\left(l_{2} / p_{1}\right) z, z_{1}=z$ and $t_{1}=-p_{1} t$ (of course, when $p_{1}>0$ there is a change in the orientation of the orbits). Then system (3.1.1) writes (we omit the subindex 1),

$$
\begin{aligned}
& \dot{y}=Y(y, z), \\
& \dot{z}=z+Z(y, z),
\end{aligned}
$$


where again $Y$ and $Z$ are polynomials in $y$ and $z$ of degree at most two. Let $z=f(y)$ be the solution of the equation $z+Z(y, z)=0$ in a neighbourhood of $(0,0)$. Then if we consider $g(y)=Y(y, f(y))$ we have

$$
\begin{array}{ll}
g(y)=\left(-1 / p_{1}\right) y^{2}+\ldots & \text { for system (II) } \\
g(y)=\left(6 / p_{1}\right) y^{2}+\ldots & \text { for system (IV) } \\
g(y)=\left(1 / p_{1}\right) y^{2}+\ldots & \text { for system (VI) }
\end{array}
$$

Therefore by Theorem DE the proposition follows.

Proposition 3.1.2. Assume that $p_{3} \neq 0$. Then the origin of system $U_{2}$ for systems (IV) and (VII) is a DE saddlemnode, and for system (V) is a DE saddle when $p_{3}>0$ and a $D E$ node when $p_{3}<0$. Furthermore the local behaviour for systems (IV) and (VII) is shown in Figure 5.

Proof: The expresion of system $U_{2}$ associated to systems (IV), (V) and (VII) is the following one

$$
\begin{aligned}
& \dot{x}=n_{1} z+X(x, z), \\
& \dot{z}=-p_{3} z+Z(x, z),
\end{aligned}
$$

where $X$ and $Z$ are polynomials in $x$ and $z$ of degree at least two. It is clear that if $p_{3} \neq 0$ then the origin of system (3.1.2) is a critical point of type DE.

By using similar arguments that in the proof of Proposition 3.1.1 we have

$$
\begin{array}{ll}
g(x)=\left(-6 / p_{3}\right) x^{2}+\ldots & \text { for the system (IV); } \\
g(x)=\left(-4 / p_{3}\right) x^{3}+\ldots & \text { for system (V); } \\
g(x)=\left(-1 / p_{3}\right) x^{2}+\ldots & \text { for system (VII); }
\end{array}
$$

Therefore by Theorem DE the proposition follows

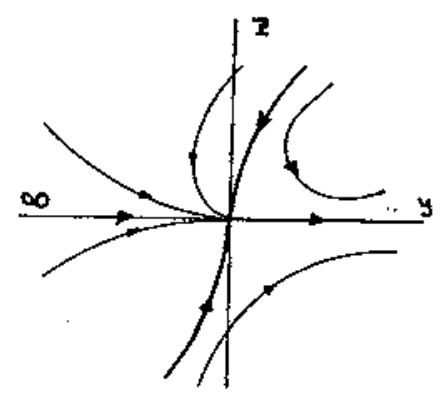

$p_{1}>0$

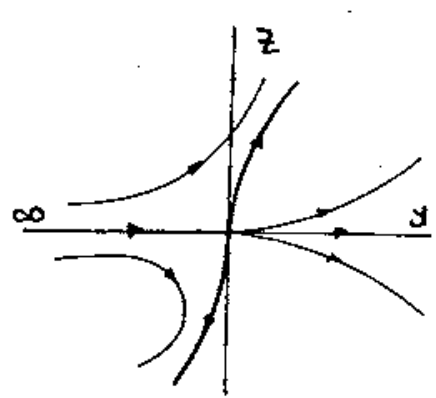

$p_{1}<0$

Figure 3. 

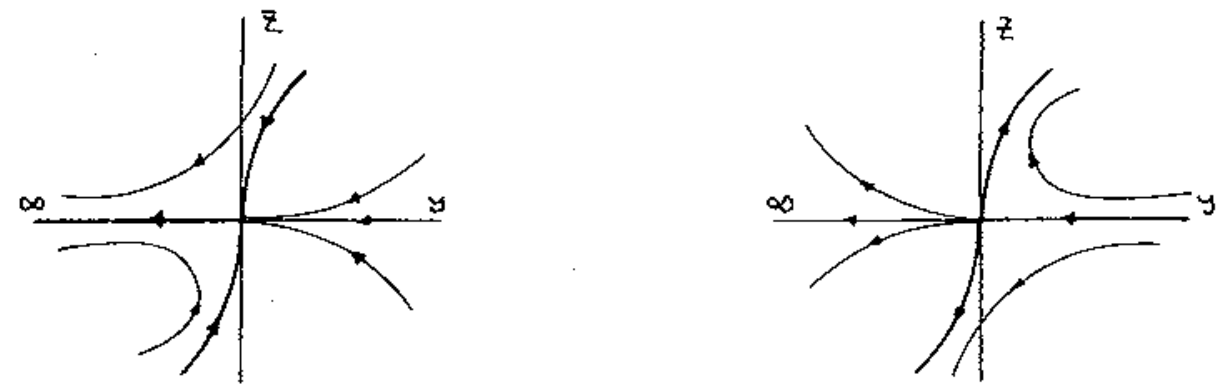

$p_{1}>0$

Figure 4.
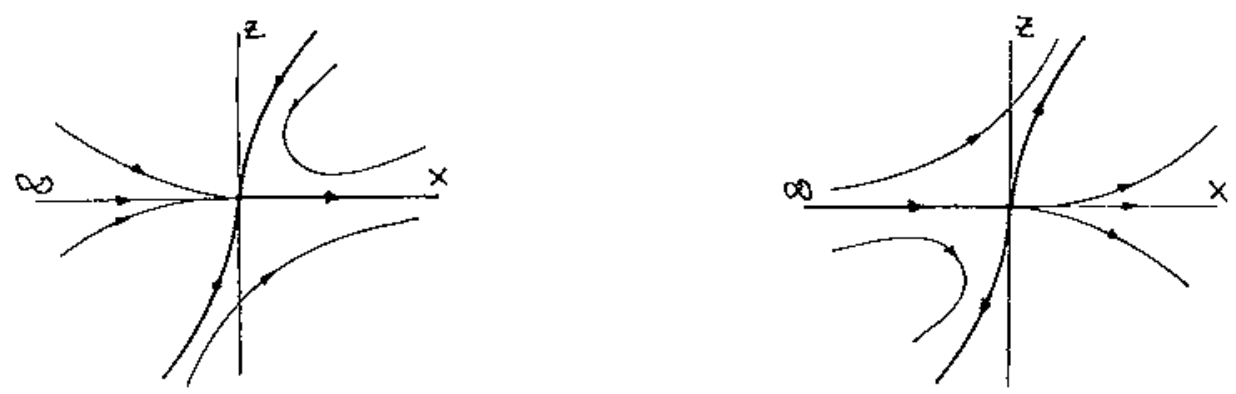

$$
p_{3}>0
$$

Figure 5.

$p_{3}<0$ 


\subsection{Non-elementary critical points in system $U_{1}$.}

In this subsection we study the local behaviour of the origin of system $U_{1}$ when it is a non-elementary critical point (NE).

Proposition 3.2.1. Assume that $p_{1}=0$ and $l_{2} \neq 0$. Then the origin of system $U_{1}$ associated to systems (II), (IV) and (VI) is a critical point of type $N E$ such that

(a) for system (II) the origin is a saddle if $p_{2}>1 / 2$, the union of a hyperbolic and elliptic sector if $p_{2}<1 / 2$, and a saddle-node if $p_{2}=1 / 2$ and $p_{3}-\left(l_{1} / l_{2}\right) \neq 0$;

(b) for systems (IV) and (VI) the origin is a saddle if $p_{2}<-3$, the union of a hyperbolic and elliptic sector if $p_{2}>-3$ and a saddle-node if $p_{2}=-3$ and $p_{3}+\left(6 l_{1} / l_{2}\right) \neq 0$.

Proof: From systme (3.1.1) (see the proof of Proposition 3.1.1) it is clear that if $p_{1}=0$ and $l_{2} \neq 0$ then the origin is a critical point of type NE. to analyse its local behaviour we use Theorem NE (see Appendix). First, we need to consider the change of time $t_{1}=l_{2} t$. Now system (3.1.1) becomes

$$
\begin{aligned}
& \dot{y}=z+Y(y, z), \\
& \dot{z}=Z(y, z),
\end{aligned}
$$

where $Y$ and $Z$ are polynomials in $y$ and $z$ of degree at least two. Let $z=f(y)$ be a solution of equation $z+Y(y, z)=0$ in the neighbourhood of $(0,0)$. Then if we consider $F(y)=Z(y, f(y))$ and $\Phi(y)=(\partial Y / \partial y+\partial Z / \partial z)(y, f(y))$ we have

$$
\begin{aligned}
& F(y)=\frac{p_{2}-1 / 2}{l_{2^{2}}} y^{3}+\left[\frac{\left(m_{2}-l_{1}\right)\left(1 / 2-p_{2}\right)}{l_{2^{3}}}+\frac{p_{3}}{l_{2^{2}}}-\frac{l_{1}}{l_{2^{3}}}\right] y^{4}+\ldots, \\
& \Phi(y)=\frac{5 / 2-p_{2}}{l_{2}} y+\ldots,
\end{aligned}
$$

for system (II);

$$
\begin{aligned}
& F(y)=\frac{-6\left(p_{2}+3\right)}{l_{2^{2}}} y^{3}+6\left[\frac{\left(m_{2}-l_{1}\right)\left(p_{2}+3\right)}{l_{2^{3}}}-\frac{p_{3}}{l_{2^{2}}}-\frac{6 l_{1}}{l_{2^{2}}}\right] y^{4}+\ldots, \\
& \Phi(y)=\frac{-\left(15+p_{2}\right)}{l_{2}} y+\ldots,
\end{aligned}
$$

for systems (IV) and (VI).

So from Theorem NE the proposition follows.

In the next results we look at the localization of the separatrices of the critical point of Proposition 3.2.1 with respect to the infinity (the equator of the Poincaré sphere). 
Lemma 3.2.2. Assume that the origin of system $U_{1}$ associated to systems (II), (IV) and (VI) is a saddle of type NE. Then its local behaviout is shown in Figure 6 .

Proof: We know that the equator of the Poincare sphere corresponds to $y$ axis in system $U_{1}$. So, on $z=0$ the system goes over to

$$
\begin{array}{lll}
\dot{y}=y^{2}-y^{4}, & \dot{z}=0 & \text { for system (II) } \\
\dot{y}=-6 y^{2}, & \dot{z}=0 & \text { for system (IV) } \\
\dot{y}=-y^{2}\left(6+y^{2}\right), & \dot{z}=0 & \text { for system (VI) }
\end{array}
$$

Then by considering the distinct possibilities of a saddle of type NE we are done.

Proposition 3.2.3. Assume that the origin of system $U_{1}$ associated to systems (II), (IV) and (VI) is the union of a hyperbolic and elliptic sector of type $N E$.

(a) For system (II) its local behaviour is shown in

$\begin{array}{lllll}\text { Figure } 7 .(1) & \text { if } & -3 / 2 \leq p_{2} \leq 1 / 2 & \text { and } & l_{2}>0 \\ \text { Figure } 7 .(2) & \text { if } & -3 / 2 \leq p_{2} \leq 1 / 2 & \text { and } & l_{2}<0 \\ \text { Figure } 7 .(3) & \text { if } & p_{2}<-3 / 2 & \text { and } & l_{2}>0 \\ \text { Figure } 7 .(4) & \text { if } p_{2}<-3 / 2 & \text { and } & l_{2}<0\end{array}$

(b) For systems (IV) and (VI) its local behaviour is shown (reversing the orientation of the orbits) in

$\begin{array}{llll}\text { Figure 7.(2) } & \text { if }-3 \leq p_{2} \leq 9 & \text { and } & l_{2}>0 \\ \text { Figure 7.(1) } & \text { if }-3 \leq p_{2} \leq 9 & \text { and } & l_{2}<0 \\ \text { Figure 7.(4) } & \text { if } p_{2}>9 & \text { and } & l_{2}>0 \\ \text { Figure 7.(3) } & \text { if } p_{2}>9 & \text { and } & l_{2}<0\end{array}$

Proof: To study the different localizations of the separatrices of the critical point $(0,0)$ os system $U_{1}$, we apply to this system two successive changes of variables of the form $y=y, z=w_{1} y$ and $y=y, w_{1}=w y$; i.e. two successive blow up's. Therefore, system $U_{1}$ is equivalent (omiting a common factor $y$ ) to

$$
\begin{aligned}
& \dot{y}=y+l_{2} y w+y^{2} Y(y, w), \\
& \dot{w}=\left[(-3 / 2)-p_{2}\right] w-2 l_{2} w^{2}+y w W(y, w),
\end{aligned}
$$

for system (II), and to

$$
\begin{aligned}
& \dot{y}=-6 y+l_{2} y w+y^{2} Y^{\prime}(y, w), \\
& \dot{w}=\left(9-p_{2}\right) w-2 l_{2} w^{2}+y w W^{\prime}(y, w),
\end{aligned}
$$

for systems (IV) and (VI), where $Y, W, Y^{\prime}$ and $W^{\prime}$ are polynomials in $y$ and $w$ of degree at least one. 

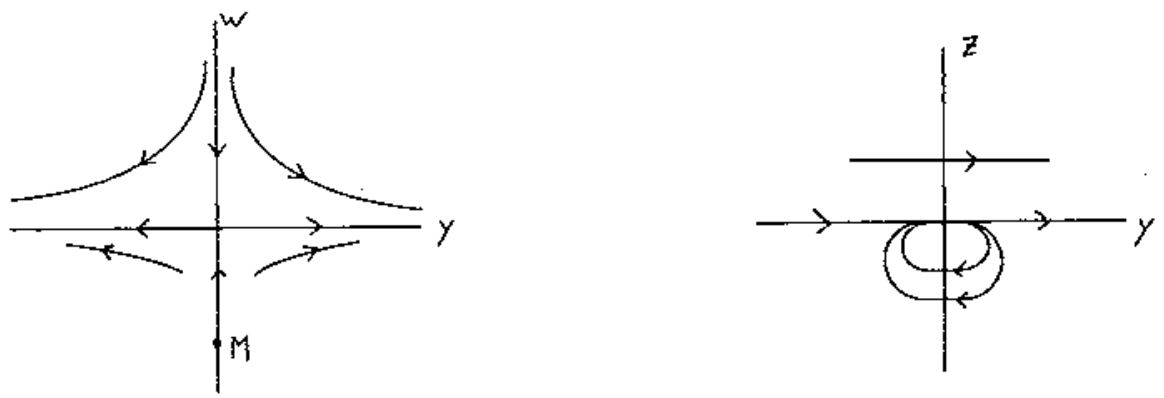

Figure 9.
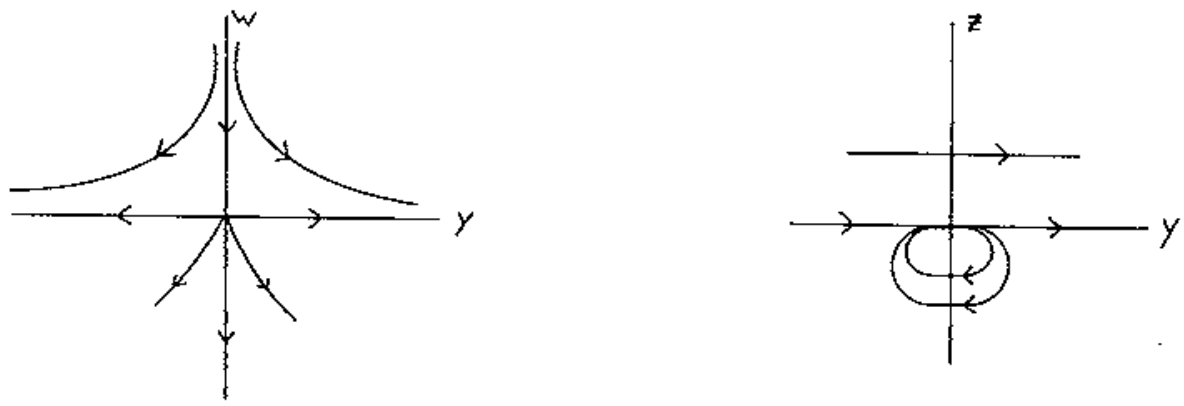

Figure 10.

Proposition 3.2.4. Assume that the origin of system $U_{1}$ associated to systems (II), (IV) and (VI) is a saddle-node of type NE. Then its local behaviour is such that two separatrices of the same hyperbolic sector are at infinity (see Figure 11.(1), for example). Moreover, sufficient conditions for all the possibilities for system (II) are shown in

Figure 12.(1) if $p_{2}=1 / 2,\left(l_{1} / l_{2}\right)-p_{3}<0$ and $l_{2}>0$;

Figure 12.(2) if $p_{2}=1 / 2,\left(l_{1} / l_{2}\right)-p_{3}<0$ and $l_{2}<0$;

Figure 12.(3) if $p_{2}=1 / 2,\left(l_{1} / l_{2}\right)-p_{3}>0$ and $l_{2}>0$;

Figure 12.(4) if $p_{2}=1 / 2,\left(l_{1} / l_{2}\right)-p_{3}>0$ and $l_{2}<0$;

and for systems (IV) and (VI) are shown (reversing the orientation of the orbits) in

Figure 12.(2) if $p_{2}=-3, p_{3}+\left(6 l_{1} / l_{2}\right)<0$ and $l_{2}>0$;

Figure 12.(1) if $p_{2}=-3, p_{3}+\left(6 l_{1} / l_{2}\right)<0$ and $l_{2}<0$;

Figure 12.(4) if $p_{2}=-3, p_{3}+\left(6 l_{1} / l_{2}\right)>0$ and $l_{2}>0$;

Figure 12.( 9$)$ if $p_{2}=-3, p_{3}+\left(6 l_{1} / l_{2}\right)>0$ and $l_{2}<0$. 
Proof: From the proof of Lemma 3.2.2 we know that only the Figures 11.(1) and 11.(2) can occur. Furthermore, from Proposition 3.2.1 if the origin is a saddle-node of type NE it is necessary that $p_{2}=1 / 2$ (resp. $p_{2}=-3$ ) for system (II) (resp. systems (IV) and (VI)). Then systems (3.2.1) and (3.2.2) of the proof of Proposition 3.2.3 write now in the form

$$
\begin{aligned}
& \dot{y}=y+l_{2} y w+y^{2} Y(y, w), \\
& \dot{w}=-2 w-2 l_{2} w^{2}+y w W(y, w),
\end{aligned}
$$

and

$$
\begin{aligned}
& \dot{y}=-6 y+l_{2} y w+y^{2} Y^{\prime}(y, w), \\
& \dot{w}=12 w-2 l_{2} w^{2}+y w W^{\prime}(y, w),
\end{aligned}
$$

respectively.

System (3.2.1') has exactly two critical points on the $w$-axis. From Theorems $\mathrm{E}$ and $\mathrm{DE}(0,0)$ is a saddle and $M=\left(0,-1 / l_{2}\right)$ is a critical point of type $\mathrm{E}$ or $\mathrm{DE}$. By similar arguments that in Case 2 of the proof of Proposition 3.2.3 we have for system (II) that only Figure 11.(1) can occur. Again, by using similar arguments to the proof of Proposition 3.2.3, we can obtain all the possibilities for system (II).

The proof for system (3.2.2') follows similary.

3.3. Non-elementary critical points in system $U_{2}$.

This subsection deals with the local behaviour of the origin of system $U_{2}$ when it is a non-elementary critical point (NE).

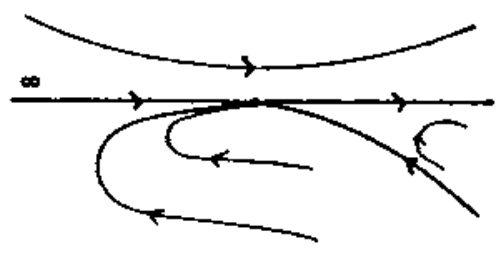

(1)

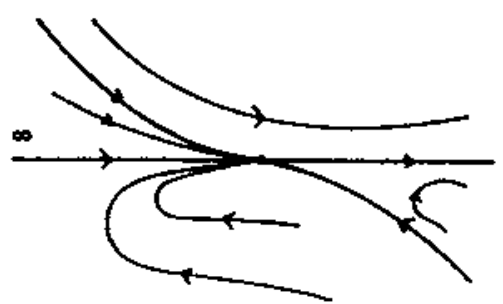

(2) 


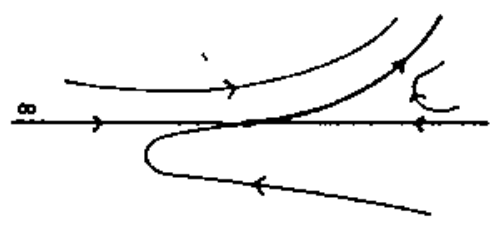

(3)

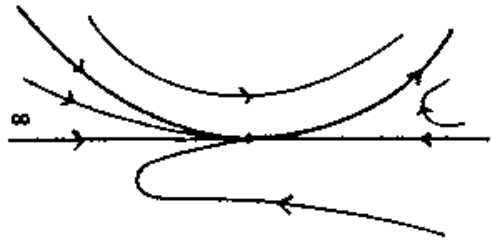

(4)

Figure 11. The possible saddle-nodes of type NE on the equator of $S^{2}$. In fact, only configuration (1) is possible (we can reverse the orientation of the orbits).

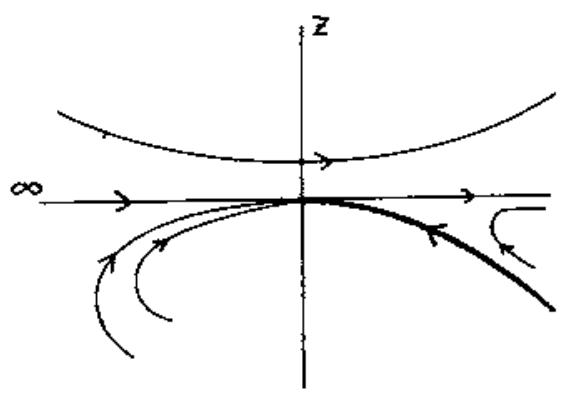

(1)

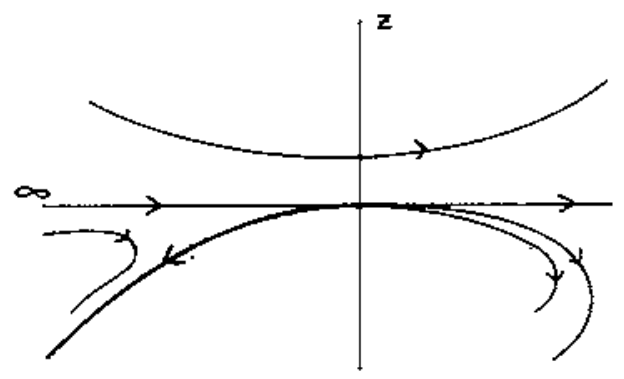

(3)

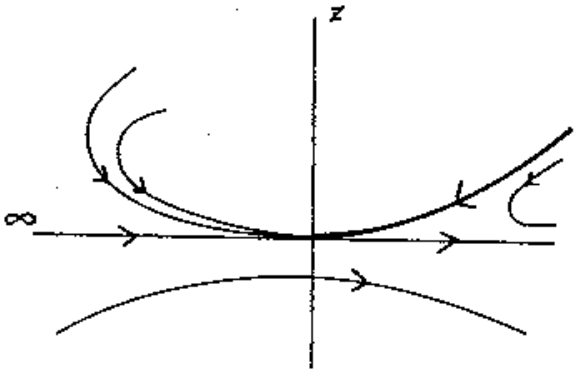

(2)

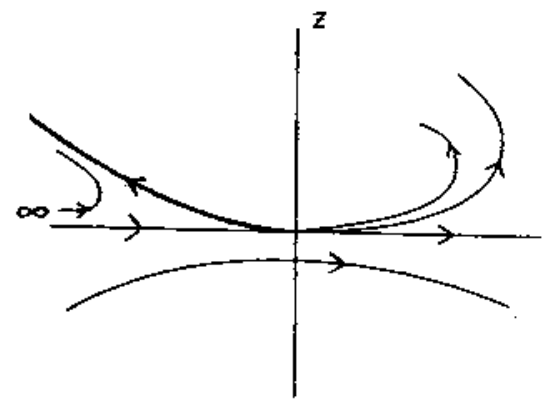

(4)

Figure 12. 
Proposition 3.3.1. Assume that $p_{3}=0$ and $n_{1} \neq 0$. Then the origin of system $U_{2}$ associated to systems (IV), (V) and (VII) is a critical point of type $N E$ such that

(a) for system (IV) (Tesp. system (VII)) the origin is a saddle if $p_{2}>3$ (resp. $p_{2}>0$ ), the union of a hyperbolic and elliptic sector if $p_{2}<3$ (resp. $p_{2}<0$ or $\left.p_{2}=p_{1}=0\right)$, and a saddle-node if $p_{2}=3$ and $p_{1}-\left(6 n_{2} / n_{1}\right) \neq 0$ (resp. $p_{2}=0$ and $\left.p_{1} \neq 0\right)$;

(b) for system (V) the origin is a saddle-node if $p_{2} \neq 0$, a saddle if $p_{2}=$ 0 and $p_{1}>2$, and a node if $p_{2}=0$ and $p_{1}<2$ (which is attractor when $\left(14-p_{1}\right) / n_{1}<0$ and repellor when $\left.\left(14-p_{1}\right) / n_{1}>0\right)$.

Proof: From system (3.1.2) (see the proof of Proposition 3.1.2) it is clear that if $p_{3}=0$ and $n_{1} \neq 0$ then the origin is a critical point of type NE. To analyse its local behaviour we use Theorem NE.

Now as in the proof of Proposition 3.2.1 we consider the functions:

$$
\begin{aligned}
& F(x)=\frac{6\left(p_{2}-3\right)}{n_{1} 2} x^{3}+6\left[\frac{\left(m_{1}-n_{2}\right)\left(3-p_{2}\right)}{n_{1}^{3}}+\frac{p_{1}}{n_{1^{2}}}-\frac{6 n_{2}}{n_{1^{3}}}\right] x^{4}+\ldots, \\
& \Phi(x)=\frac{15-p_{2}}{n_{1}} x+\ldots,
\end{aligned}
$$

for system (IV);

$$
\begin{aligned}
& F(x)=\frac{4 p_{2}}{n_{12}} x^{4}+4\left[\frac{p_{2}\left(n_{2}-m_{1}\right)}{n_{1}}+p_{1}-2\right] x^{5}+\ldots, \\
& \Phi(x)=-\frac{p_{2}}{n_{1}} x+\frac{14-p_{1}}{n_{1}} x^{2}+\ldots,
\end{aligned}
$$

for system $(V)$;

$$
F(x)=-\frac{p_{2}}{n_{1^{2}}} x^{5}+\frac{A p_{2} n_{1}+p_{1}}{n_{1^{2}}} x^{6}+\frac{B p_{2} n_{1}+C p_{1} n_{1}-1}{n_{1^{2}}} x^{7}+\ldots,
$$

where $A, B$ and $C$ are constants,

$$
\Phi(x)=-\frac{p_{2}}{n_{1}} x-\frac{p_{1}}{n_{1}} x^{2}+\frac{5}{n_{1}} x^{3}+\ldots,
$$

for system (VII).

Then by Theorem NE the proposition follows.

In the next results we study the localization of the separatrices of the critical point of Proposition 3.3.1 with respect to the infinity. 
Lemma 3.3.2. Assume that the origin of system $U_{2}$ associated to systems (IV), (V) and (VII) is a saddle of type NE. Then its local behaviour is shown in Figure 6 for systems (IV) and (VII), and in Figure 19 for system (V).

Proof: As in the proof of Lemma 3.2.2 system $U_{2}$ on $z=0$ becomes

$$
\begin{array}{lll}
\dot{x}=6 x^{2}, & \dot{z}=0 & \text { for system (IV); } \\
\dot{x}=4 x^{3}, & \dot{z}=0 & \text { for system (V); } \\
\dot{x}=x^{4}, & \dot{z}=0 & \text { for system (VII); }
\end{array}
$$

In short, by considering the distinct possibilities of a saddle of type NE we are done.
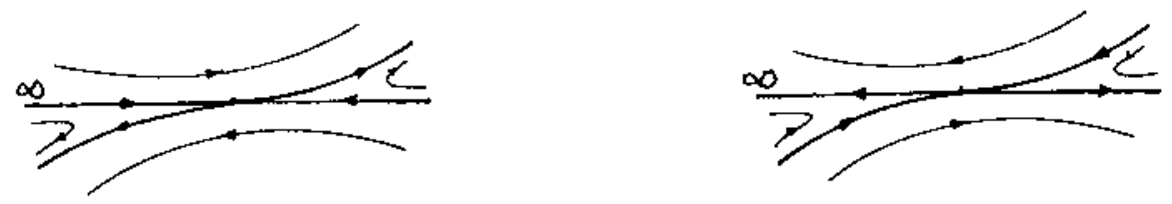

Figure 13 (we can reverse the orientation of the orbits).

\section{Proposition 3.3.3.}

(a) Assume that the origin of system $U_{2}$ associated to system (IV) is the union of a hyperbolic and elliptic sector of type NE. Then its local behaviour is shown in

$\begin{array}{llll}\text { Figure 7.(1) } & \text { if }-9 \leq p_{2} \leq 3 & \text { and } & n_{1}>0 \\ \text { Figure 7.(2) } & \text { if }-9 \leq p_{2} \leq 3 & \text { and } & n_{1}<0 \\ \text { Figure 7.(3) } & \text { if } p_{2}<-9 & \text { and } & n_{1}>0 \\ \text { Figure 7.(4) if } p_{2}<-9 & \text { and } & n_{1}<0\end{array}$

(b) If $p_{2}<0$ then the origin of system $U_{2}$ associated to system (VII) is the union of a hyperbolic and elliptic sector of type NE. In this case, its local behaviour is shown in

$$
\begin{aligned}
& \text { Figure 7.(3) if } p_{2}<0 \text { and } n_{1}>0 \text {; } \\
& \text { Figure 7.(4) if } p_{2}<0 \text { and } n_{1}<0 .
\end{aligned}
$$

Proof: By using similar arguments to the proof of Proposition 3.2.3, we apply to system $U_{2}$ two successive changes of variables $x=x, z=w_{1} x$ and $x=x$, $w_{1}=w x$. Therefore, system $U_{2}$ is equivalent (omitting a common factor $x$ ) to

$$
\begin{aligned}
& \dot{x}=6 x+n_{1} x w+x^{2} X(x, w), \\
& \dot{w}=-\left(9+p_{2}\right) w-2 n_{1} w^{2}+x w W(x, w),
\end{aligned}
$$


for system (IV), and to

$$
\begin{aligned}
& \dot{x}=n_{1} x w+x^{2} X^{\prime}(x, w), \\
& \dot{w}=-p_{2} w-2 n_{1} w^{2}+x w W^{\prime}(x, w),
\end{aligned}
$$

for system (VII), where $X, W, X^{\prime}$ and $W^{\prime}$ are polynomials in $x$ and $z$ of degree at least one.

The study of system (3.3.1) follows in a similar way to the study of system (3.2.1) in the proof of Proposition 3.2.3. In short, (a) follows.

If $p_{2}<0$ system (3.3.2) has exactly two critical points on the $w$-axis. From Theorems $\mathrm{E}$ and DE, $(0,0)$ is an unstable node and $M=\left(0,-p_{2} /\left(2 n_{1}\right)\right)$ is a saddle. So, from Figure 8 , (b) follows.

We know from the proof of Proposition 3.3.1 that the origin of system $U_{2}$ for system (IV) can be the union of a hyperbolic and elliptic sector if $p_{2}=3$ plus other conditions. It follows from the above proof that this situation is also contained in Proposition 3.3.3. Moreover, from Proposition 3.3.1, if $p_{2}=p_{1}=0$ then the origin of system $U_{2}$ for system (VII) is the union of a hyperbolic and elliptic sector. This case will be studied in Section 10.

\section{Proposition 3.3.4.}

(a) Assume that the origin of system $U_{2}$ associated to system (IV) is a saddle-node of type NE. Then its local behaviour is such that two separatrices of the same hyperbolic sector are at infinity (see Figure 11.(1), for example). Moreover, sufficient conditions for all the possibilities are shown in

$$
\begin{array}{lllll}
\text { Figute 12.(1) if } p_{2}=3, & \left(6 n_{2} / n_{1}\right)-p_{1}<0 & \text { and } & n_{1}>0 \\
\text { Figure 12.(2) } & \text { if } p_{2}=3, & \left(6 n_{2} / n_{1}\right)-p_{1}<0 & \text { and } & n_{1}<0 \\
\text { Figure 12.(3) if } p_{2}=3, & \left(6 n_{2} / n_{1}\right)-p_{1}>0 & \text { and } & n_{1}>0 \\
\text { Figute 12.(4) if } p_{2}=3, & \left(6 n_{2} / n_{1}\right)-p_{1}>0 & \text { and } & n_{1}<0
\end{array}
$$

(b) If $p_{2} \neq 0$ then the origin of system $U_{2}$ associated to system (V) is a saddle-node. In this case its local behaviout is shown in

$$
\begin{array}{lllll}
\text { Figure 14.(1) if } & p_{2}<0 & \text { and } & n_{1}>0 \\
\text { Figure 14.(2) if } & p_{2}<0 & \text { and } & n_{1}<0 \\
\text { Figure 14.(3) if } & p_{2}>0 & \text { and } & n_{1}>0 ; \\
\text { Figure 14.(4) if } & p_{2}>0 & \text { and } & n_{1}<0 .
\end{array}
$$

Proof: Statement (a) follows analogously to the proof of Proposition 3.2.4.

From the proof of Lemma 3.3.2 we know that only the Figures 11.(3) and 11.(4) can occur. Now we apply to systern $U_{2}$ the same change of variables that in the proof of Proposition 3.3.3 and we obtain

$$
\begin{aligned}
& \dot{x}=4 x^{2}+n_{1} x w+x^{2} X(x, w), \\
& \dot{w}=-p_{2} w-2 n_{1} w^{2}-6 x w+x w W(x, w),
\end{aligned}
$$


for system (V), where $X, W$ are polynomials in $x$ and $w$ of degree at least one.

System (3.3.3) has exactly two critical points on the $w$-axis if $p_{2} \neq 0$. From Theorems $E$ and $D E,(0,0)$ is a saddle-node and $M=\left(0,-p_{2} /\left(2 n_{1}\right)\right)$ is a saddle. Furthermore, the saddle-node has the two hyperbolic sectors either to the right or to the left of the invariant $w$-axis. So, from Figure 15 (where we suppose $p_{2}<0$ and $n_{1}>0$ ) statement (b) follows.

We know from the proof of Proposition 3.3.1 that the origin of system $U_{2}$ for system (V) could be a saddle-node if $p_{2}=0$ plus others conditions. This case will be studied in Section 8 .

Note that we do not consider the case that the origin of system $U_{2}$ associated to system (VII) is a NE saddle-node. We shall prove in Section 10 that this case cannot occur for chordal cubic systems.

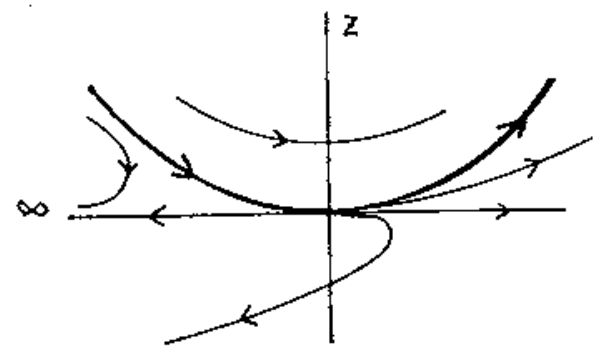

(1)

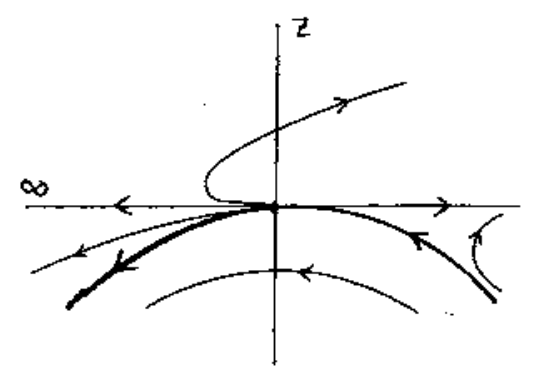

(3)

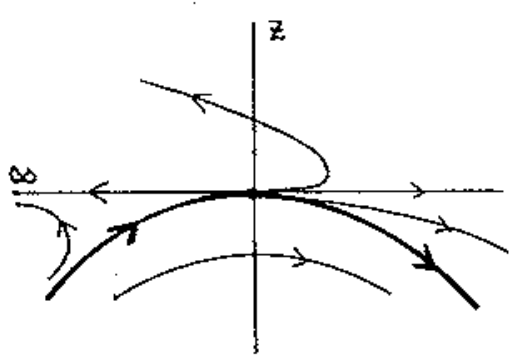

(2)

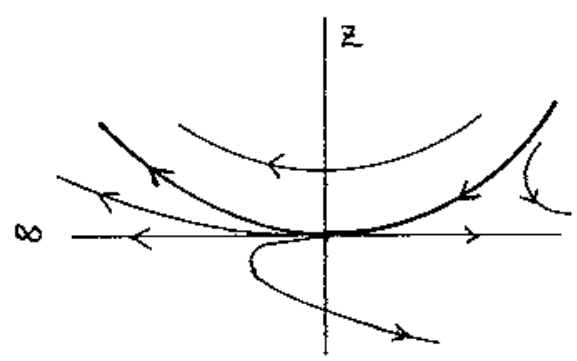

(4)

Figure 14. 

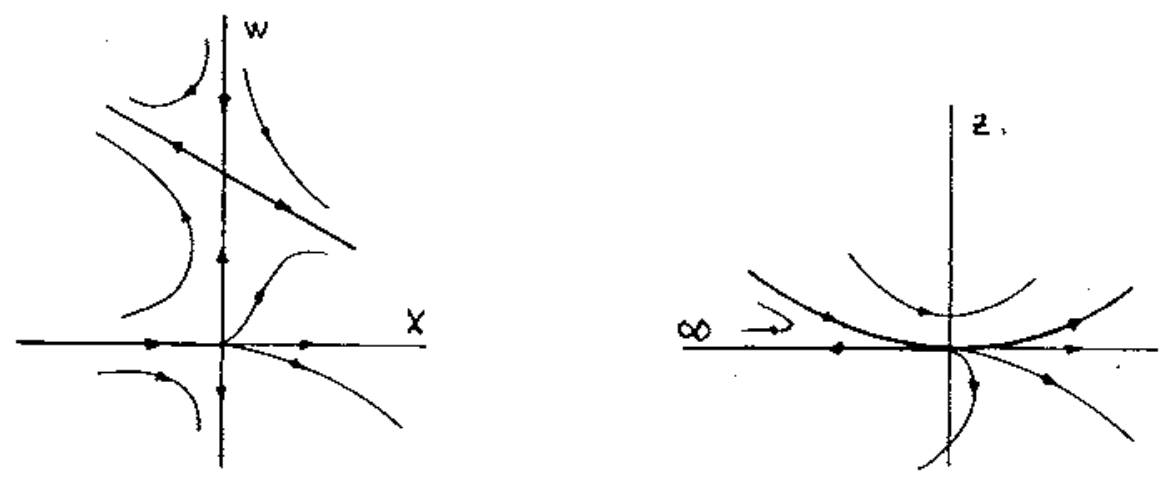

Figure 15.

\section{System (I)}

In this section we determine all the possible phase portraits of $\operatorname{CCS(I).~Also~}$ we realize these phase portraits.

\subsection{Critical points.}

First we study the critical points at infinity of system (I).

Remark. 4.1.1. From Appenix $A$ of [6], to study the critical points at infinity it is enough to study systems $U_{1}$ and $U_{2}$. Moreover, since the infinite critical points of a CS appear in diametrically opposite pairs (each critical point of the same pair has the same local behaviour), we only look at the critical points of system $U_{1}$ with $z=0$, and at the origin of system $U_{2}$.

An easy computation allows to prove the next result (see Appendix $A$ of $[6]$ ).

Lemma 4.1.1. System $U_{1}$ associated to system (I) has four critical points on $z=0:(\mu, 0),(-\mu, 0),(1 / \mu, 0)$ and $(-1 / \mu, 0)$.

We denote by $y_{1}, y_{2}, y_{3}$ and $y_{4}$ the values $\mu, 1 / \mu,-\mu$ and $-1 / \mu$, respectively.

The next two lemmas follow from Appendix $A$ of $[6]$ and Appendix I.

Lemma 4.1.2. The critical point $\left(y_{k}, 0\right)$ of system $U_{1}$ associated to system (I) is of type $E$ if $P_{3}\left(1, y_{k}\right) \neq 0$ and of type $D E$ if $P_{3}\left(1, y_{k}\right)=0$. In this second case, if the critical point is a saddle-node, then it is of type DE1 (see Appendix I).

Lemma 4.1.3. The vector field on the equator of the Poincaré sphere of system (I) is shown in Figure 16. 


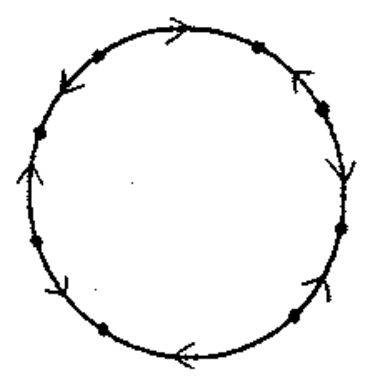

Figure 16.

We shall say that system (I) has index configuration $(i, j, k, l)$ if the four pairs of infinite critical points have indices $i, j, k$ and 1 .

From Theorems E, DE and Poincaré-Hopf Theorem (see Appendix I) we have that a $\operatorname{CCS}(I)$ has only two possible index configurations, $(1,1,-1,0)$ and $(1,0,0,0)$.

\subsection{Topological phase portraits.}

In this subsection we study all the possible topological phase portraits of a $\operatorname{CCS}(\mathrm{I})$. To do that we analyse all the distinct index configurations.

Configuration $(1,1,-1,0)$.

From Lemma 4.1.2 and Theorems $\mathrm{E}$ and $\mathrm{DE}$, it follows that the infinite critical points are two nodes, one saddle and one saddle-node of type DE1.

Proposition 4.2.1. The phase portrait of a CCS(I) with index configuration $(1,1,-1,0)$ is homeomorphic (except, perhaps the orientation) to one of phase portraits $1,2,3$ or 4 .

Proof: By using symmetries, rotations and changes of sign in the variable $t$ (if it is necessary), we obtain that any $\operatorname{CCS}(I)$ with index configuration $(1, I$, $-1,0$ ) has a behaviour at infinity equivalent to one of the behaviours that are shown in Figure 17.

By looking at Figure 17.(1) we have that there are three possible phase portraits with this behaviour in a neighbourhood of the infinity, phase portraits 1, 2 and 3. Moreover from Figure 17.(2) we have that there is only one possible phase portrait with this behaviour in a neighbourhood of the infinity, the phase portrait 4. 


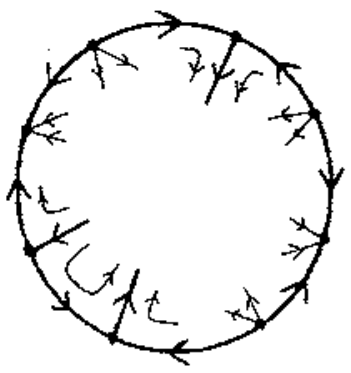

(1)

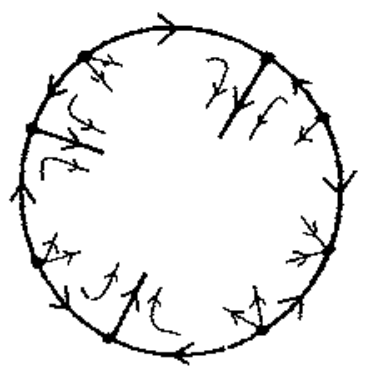

(2)

Figure 17 .

Configuration $(1,0,0,0)$.

From Theorems $E$ and $D E$ and Lemma 4.1.2 it follows that the infinite critical points are one node and three saddle-nodes of type DE1.

Proposition 4.2.2. The phase portrait of a CCS(I) with index configuration $(1,0,0,0)$ is homeomorphic (except, perhaps the orientation) to one of phase portraits $5,6,7,8$, and 9 .

Proof: By using similar arguments to those of the proof of Proposition 4.2.1 we obtain that any $\operatorname{CCS}(\mathrm{I})$ with index configuration $(1,0,0,0)$ has a behaviour at infinity as in Figure 18.

Figures 18.(1) and 18.(2) determine phase portraits 5 and 6 ; respectively. Figure 18.(3) determines phase portraits 7,8 and 9.

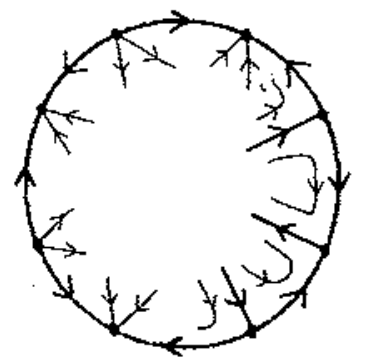

(1)

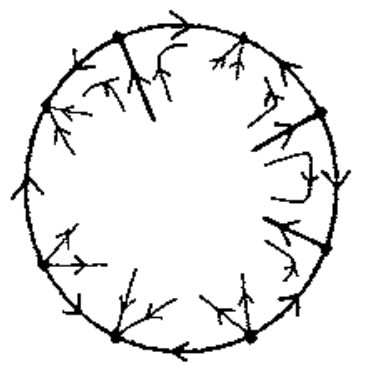

(2)

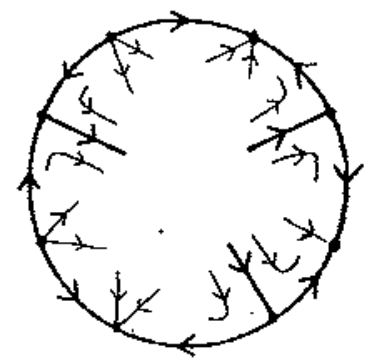

(3)

Figure 18. 


\subsection{Realizations.}

In this subsection we shall give $\mathrm{CCS}(\mathrm{I})$ that realize each phase portrait found in Subsection 4.2.

\section{Phase portraits 1,2 and 3.}

From the proof of Proposition 4.2.1 we know that phase portraits 1, 2 and 3 have the same behaviour at infinity. Now we consider the following family of CS:

$$
\dot{x}=6(y+(1 / 2) x)\left(4 y^{2}-2 x y+x^{2}\right),
$$

$$
\dot{y}=d-2 x-4 y+(53 / 4) x^{2}-16 x y+5 y^{2}+6 x(y+(1 / 2) x)(17 y-8 x),
$$

with $d>0$. All the systems of this family are of type (I) of Theorem 2.3 with $\mu=2$.

The next result is easy to prove.

Lemma 4.3.1. If $d>0$ then system (4.3.1.d) is a $\operatorname{CCS}(1)$.

Now we study the infinite critical points of this family of systems.

\section{Lemma 4.3.2.}

(a) System $U_{1}$ associated to system $(4.9,1, d)$ has four critical points on $z=0,(2,0)$ is an attractor node, $(1 / 2,0)$ is a saddle, $(-1 / 2,0)$ is a saddlenode of type DEI and $(-2,0)$ is a repellor node.

(b) The behaviour at infinity of systems (4.3.1.d) is shown in Figure 19.

Proof: By Lemma 4.1.1 we know that $(2,0),(1 / 2,0),(-1 / 2,0)$ and $(-2,0)$ are the critical points of system $U_{1}$ on $z=0$. From Appendix $A$ of [6] and Appendix I we obtain that $(2,0)$ is an attractor node, $(1 / 2,0)$ is a saddle, $(-2,0)$ is a repellor node and $(-1 / 2,0)$ is of type DE. Since system (4.3.1.d) is chordal (see Lemma 4.3.1), from Poincaré-Hopf Theorem we have that $(-1 / 2,0)$ has index zero. so, from Theorem $\mathrm{DE}(-1 / 2,0)$ is a saddle-node and from Lemma 4.1.2 it is of type DE1. Hence we obtain (a).

Statement (b) follows by applying Theorem DE to determine the localization of the separatrix of the saddle-node not contained at infinity, and using statement (a). 


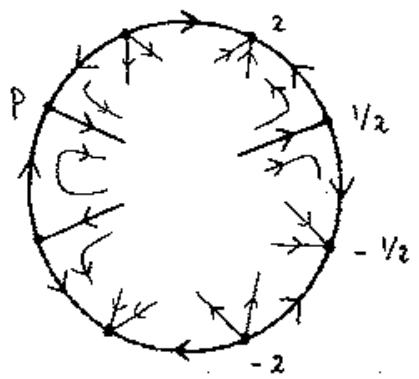

Figure 19.

\section{Proposition 4.3.3.}

(a) System (4.3.1.d) with $d=1 / 2$ realizes phase portroit 3 .

(b) System (4.3.1.d) with $d=21$ realizes phase portrait 1 .

(c) System (4.3.1.d) for some $d \in(1 / 2,21)$ realizes phase portiait 2 .

Proof: From Figure 19, to realize phase portrait 3 it is enough to find a value $d$, such that system (4.3.1.d) verifies that the separatrix not contained at infinity of the saddle-node $p$ (see Figure 19) goes to the critical point $(-1 / 2,0)$. To do that we consider the straight line $f(x, y)=y+x / 2-1 / 4=0$ and we try to impose that it is a solution. This is equivalent to show that grad $f(x, y) \cdot(\dot{x}, \dot{y})=\dot{y}+\dot{x} / 2=0$ on $f(x, y)=0$. Since $\dot{y}+\dot{x} / 2=d-1 / 2$, system (4.3.1.d) with $d=1 / 2$ has $f(x, y)=0$ as a solution. Then, from Lemmas 4.3 .1 and 4.3 .2 statement (a) follows.

To realize phase portrait 1 we look at the vector field of system (4.3.1.d) on the straight line $g(x, y)=y-2 x=0$. Since $\operatorname{grad} g(x, y) \cdot(\dot{x}, \dot{y})=(5 / 4) x^{2}-$ $10 x+d$ on $g(x, y)=0$, if $d=21$ the straight line is without contact points and the orbits crosses it from the half-plane $y-2 x<0$ to the other one in forward time. Therefore, from Figure 19 the separatrix of the saddle $(1 / 2,0)$ must come from the node $(-2,0)$. So from Lemmas 4.3 .1 and 4.3 .2 statement, (b) is proved.

Now we shall realize the phase portrait 2 . Any system of the family (4.3.1.d) is a CCS(I) with the behaviour at infinity shown in Figure 19 (see Lemma 4.3.2). Moreover if $d=1 / 2$ and $d=21$ the corresponding systems realize phase portraits 3 and 1 , respectively. So, from the continuity of the solutions with respect to parameters there exists an intermediate value of $d$ that realizes phase portrait 2 .

More exactly, we consider the interval $[a, b]$ with $0<a<1 / 2$ and $b>21$. Since (2.0) is an attractor node of system (4.3.1.d) for all $d \in[a, b]$ (see Lemma 4.3.2) we clain that there exists a neighbourhood $U$ of $(2,0)$ such that any orbit in $U$ goes to the critical point $(2,0)$. Now we consider the set $M$ of values $d \in[a, b]$ such that for the corresponding system (4.3.1.d) the separatrix of the saddle-node $p$ goes to $(2,0)$. We shall prove that $M$ is an open set. 
From the theorems of continuous dependency of the solutions with respect to parameters and initial conditions, if $d_{0} \in M$, then there is an interval $V=$ $\left(d_{0}-\varepsilon, d_{0}+\varepsilon\right)$ such that, for each system (4.3.1.d) with $d \in V$, the corresponding separatrix of the saddle-node $p$ (out of a neighbourhood - independent on $d$ of this critical point $p$ ) goes into the neighbourhood $U$ in a finite time. Hence $M$ is open.

Similarly, we have that the set $N$, of values $d \in[a, b]$ such that for the corresponding system (4.3.1.d) the separatrix of the saddle-node $p$ goes to $(-1 / 2,0)$, is an open set.

Since $1 / 2 \in N, 21 \in M, M \cap N=\phi$ and $[a, b]$ is connected, there exists a $d^{*} \in[1 / 2,21]$ such that $d^{*} \notin M \cup N$. That is, for system (4.3.1.d) with $d=d^{*}$ the separatrix of the saddle-node $p$ cannot go neither $(2,0)$ nor $(-1 / 2,0)$. So, it must go to the critical point $(1 / 2,0)$ and therefore system (4.3.1.d) with $d=d^{*}$ realizes phase portrait 2 .

Phase portraits 4,5 and 6.

From the proofs of Propositions 4.2.1 and 4.2.2 it follows that the phase portraits 4,5 and 6 are such that the behaviour at infinity determines completely the phase portrait.

Proposition 4.3.4. System

$$
\begin{aligned}
& \dot{x}=6 \mu^{5} x^{3}+6 \mu^{2} y^{3}, \\
& \dot{y}=1+x^{2}-6 \mu^{2} x^{3}+6 \mu^{5} x^{2} y+6\left(1+\mu^{4}\right) x y^{2},
\end{aligned}
$$

with $\mu>1$ is a CCS(I) and realizes phase portrait 4 .

Proof: By easy computations it follows that system (4.3.2) is a CCS(1).

From Appendices $A$ of $[6]$ and I we obtain that the four critical points at infinity (see Lemma 4.1.1) are two attractor nodes $(\mu, 0)$ and $(-1 / \mu, 0)$, a saddle $(1 / \mu, 0)$ and a critical point $(-\mu, 0)$ of type $\mathrm{DE}$. By using similar arguments to the proof of Lemma 4.3 .2 it follows that $(-\mu, 0)$ is a saddle-node of type DE1. Therefore, we have that the behaviour at infinity of system (4.3.2) is given by Figure 17.(2) reversing the orientation of the orbits. Hence the proposition follows.

Proposition 4.3.5. System

$$
\dot{x}=-(7 / 4) x-2 y+24(y-x / 2)(y+x / 2)(y+2 x),
$$

$$
\dot{y}=-1 / 4-(7 / 2) x-4 y+x^{2}+4 x y+4 y^{2}+48(y-x / 2)(y+x / 2)(y+2 x),
$$

is a CCS(I) and realizes phase portrait 5 . 
Proof: It is clear that system (4.3.3) is a CS of type (I) with $\mu=2$. To show that this system has no finite critical points, we write it in the form

$$
\begin{aligned}
& \dot{x}=A(x, y)+P_{3}(x, y)=0, \\
& \dot{y}=B(x, y)+Q_{3}(x, y)=0,
\end{aligned}
$$

where $P_{3}(x, y)=24(y-x / 2)(y+x / 2)(y+2 x)$. Then its finite critical points are solutions of the system

$$
\begin{aligned}
& A(x, y)+P_{3}(x, y)=0 \\
& B(x, y)-2 A(x, y)=0
\end{aligned}
$$

From direct computations $B(x, y)-2 A(x, y)=4(y+x / 2+1 / 4)(y+x / 2-1 / 4)$. So, if we denote $y_{1}=-x / 2-1 / 4, y_{2}=-x / 2+1 / 4$ and substitute these expressions in the first equation we obtain that $A\left(x, y_{1}\right)+P_{3}\left(x, y_{1}\right)=9 x^{2}+1 / 8$ and that $A\left(x, y_{2}\right)+P_{3}\left(x, y_{2}\right)=-9 x^{2}-1 / 8$. Hence system (4.3.3) is a $\operatorname{CCS}(\mathrm{I})$.

From Appendices $A$ of $[6]$ and I we have that the four critical points at infinity (see Lemma 4.1.1) are an attractor node $(2,0)$ and three critical points $(1 / 2,0),(-1 / 2,0)$ and $(-2,0)$ of type DE. To study the local behaviour of these three critical points we apply Theorem DE. By using similar arguments to the proof of Proposition 3.1.1 we obtain

$$
\begin{array}{ll}
g(z)=(4 / 135) z^{2}+\ldots & \text { for }(1 / 2,0), \\
g(z)=z^{4} / 900+\ldots & \text { for }(-1 / 2,0), \text { and } \\
g(z)=z^{2} / 160+\ldots & \text { for }(-2,0) .
\end{array}
$$

So, by Theorem DE they are saddie-nodes of type DEI and the behaviour at infinity is given by Figure 18.(1), and we are done.

\section{Proposition 4.3.6. System}

$$
\dot{x}=-(21 / 4) x+24(y-x / 2)(y+x / 2)(y+2 x),
$$

$$
\dot{y}=-1 / 4-(21 / 2) x+x^{2}-4 x y+4 y^{2}+48(y-x / 2)(y+x / 2)(y+2 x),
$$

is a CCS(I) and realizes phase portrait 6 .

Proof: As in the proof of Proposition 4.3.5, we obtain that system (4.3.4) is a CCS(I). The critical points at infinity are an attractor node $(2,0)$, and three critical points $(1 / 2,0),(-1 / 2,0)$ and $(-2,0)$ of type DE. From Theorem DE we have that

$$
\begin{array}{ll}
g(z)=-z^{4} / 540+\ldots & \text { for }(1 / 2,0), \\
g(z)=-(4 / 225) z^{2}+\ldots & \text { for }(-1 / 2,0), \text { and } \\
g(z)=(5 / 288) z^{2}+\ldots & \text { for }(-2,0) .
\end{array}
$$


Lemma 5.1.1. System $U_{1}$ associated to system (II) has three critical points on $z=0,(1,0),(0,0)$ and $(-1,0)$.

The next two lemmas follow from the Poincaré compactification (see Appen$\operatorname{dix} A$ of $[6]$ and Proposition 3.1.1)

\section{Lemma 5.1.2.}

(a) The critical point $(k, 0)$ of system $U_{1}$ associated to system $(I I)$ is of type $E$ if $P_{3}(1, k) \neq 0$, and of type $D E$ if $P_{3}(1, k)=0$, where $k \in\{-1,1\}$. Furthermore if the critical point is a DE saddle-node, then it will be of type DE1 (see Appendix I).

(b) The critical point $(0,0)$ of system $U_{1}$ associated to system (II) is, of type $D E$ if $p_{1} \neq 0$; of type $N E$ if $p_{1}=0, l_{2} \neq 0$, and of type $Z$ if $p_{1}=l_{2}=0$. If it is of type DE then it is a saddle-node of type DEQ (see Appendix I).

Lemma 5.1.3. The vector field on the equator of the Poincaré sphere of system (II) is shown in Figure 22.

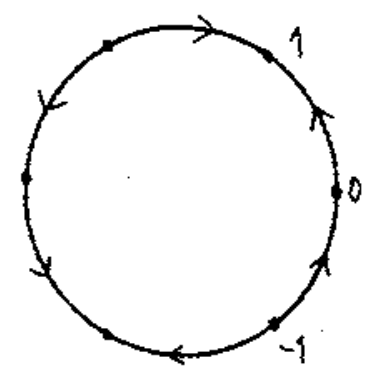

Figure 22.

We shall say that system (II) has index configuration $(i, j, k)$ if its three pairs of infinite critical points have indices $i, j$ and $k$.

From Theorems E, DE, NE and Poincaré-Hopf Theorem (see Appendix I) we have that a CCS(II) has only two possible index configurations, $(1,1,-1)$ and $(1,0,0)$.

\subsection{Topological phase portraits.}

In this subsection we study all the possible topological phase portraits of a CCS(II). First, we analyse the distinct index configurations.

Configuration $(1,1,-1)$.

From Lemma 5.1.2 and Theorems $\mathrm{E}, \mathrm{DE}$ and $\mathrm{NE}$, the critical points at infinity have two options, either they are two nodes and one saddle (Option 1), or they are one node, one saddle and the union of a hyperbolic and elliptic sector (Option 2). 
Proposition 5.2.1.

(a) The phase portrait of a CCS(II) satisfying Option 1 is homeomorphic (except, perhaps the orientation) to phase portrait 10.

(b) The phase portrait of a CCS(II) satisfying Option 2 is homeomorphic (except, perhaps the orientation) to one of phase portraits $11,12,19$ and 14 .

\section{Proof:}

(a) From Figure 22 and Theorems $\mathrm{E}$ and $\mathrm{DE},(0,0)$ must be a saddle of type NE. Then, from Lemma 3.2 .2 our CCS(II) has a behaviour at infinity given by Figure 23 . This behaviour at infinity determines phase portrait 10 .

(b) From Lemma 5.1.2 and Theorems E, DE and NE, $(0,0)$ must be the union a hyperbolic and elliptic sector. Then, from Proposition 3.2.3 and by using symmetries and rotations, our $\mathrm{CCS}$ (II) has a behaviour at infinity given by (1) or (2) of Figure 24. Figure 24.(1) determines phase portrait 11. However, Figure 24.(2) determines phase portraits 12,13 and 14.

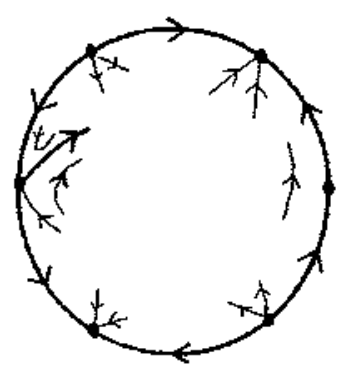

Figure 23 .

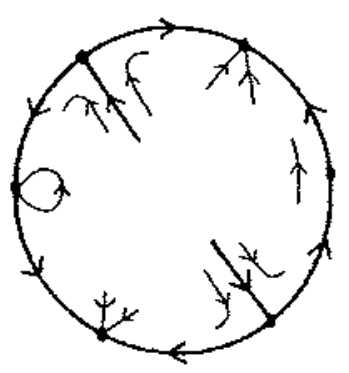

(1)

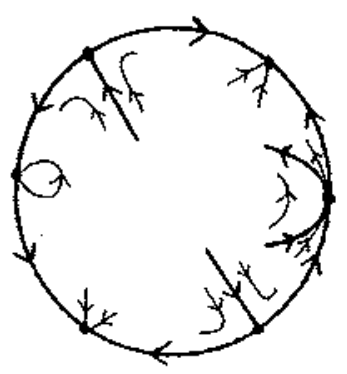

(2)

Figure 24. 
Conftguration $(1,0,0)$.

From Lemma 5.1.2 and Theorems E, DE and NE, the critical points at infinity have two options, either they are one node and two saddle-nodes (Option 1), or two saddle-nodes and the union of a hyperbolic and elliptic sector (Option 2).

\section{Proposition 5.2.2.}

(a) The phase portrait of a CCS(II) satisfying Option 1 is homeomorphic (except, perhaps the orientation) to one of phase portraits 15,16, 17, 18,19, 20, 21, 22, 23 and 24.

(b) The phase portrait of a CCS(II) satisfying Option 2 is homeomorphic (except, perhaps the orientation) to one of phase portraits $25,26,27$ and 28.

\section{Proof:}

(a) From Figure 22, $(0,0)$ must be a saddle-node, either of typc DE or NE. In the first case, from Lemma 5.1 .2 an by using symmetries and rotations, our CCS(II) has a behaviour at infinity given by Figure 25. In the second case, from Proposition 3.2.4 and by using symmetries an rotations, our CCS(II) has a behaviour at infinity like in Figure 26. Then Figures 25.(1), 26.(1), 26.(2) and 26.(4) determine phase portraits $15,19,20$ and 24, respectively. Figures 25 .(2) and 26.(3) determine phase portraits $16,17,18$, and $21,22,23$; respectively.

(b) Since $P_{3}(x, y)=p_{1} x^{3}+\left(p_{2}-1 / 2\right) x^{2} y+p_{3} x y^{2}+y^{3}$ for system (II), by Lemma 5.1.2 we have that $p_{1}=0, p_{2}=-1 / 2, p_{3}=0$ and $l_{2} \neq 0$. So, from Proposition 3.2.3 and by using symmetries and rotations, our CCS(II) has the behaviour at infinity shown in Figure 27. Figures 27.(1) and 27.(2) yieid phase portraits 25 and 26, respectively; and Figure 27.(3) yields phase portraits 27 and 28 .

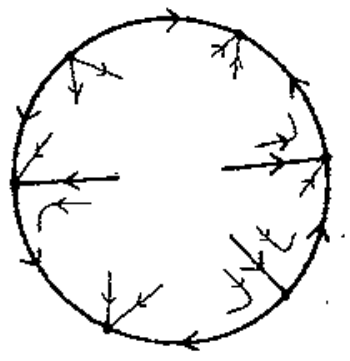

(1)

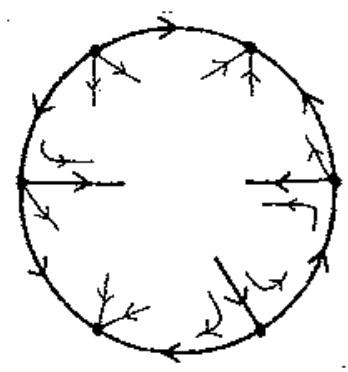

(2)

Figure 25. 


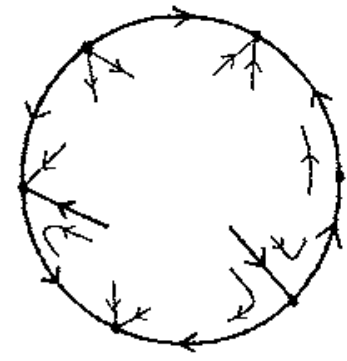

(1)

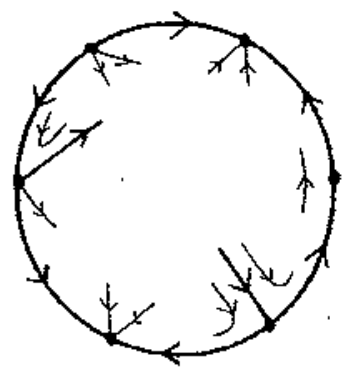

(3)

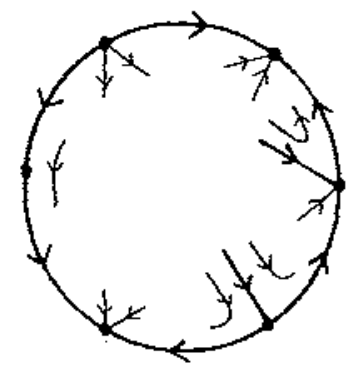

(2)

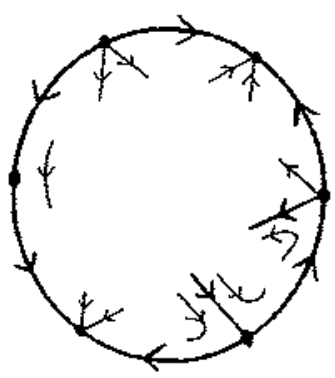

(4)

Figure 26.

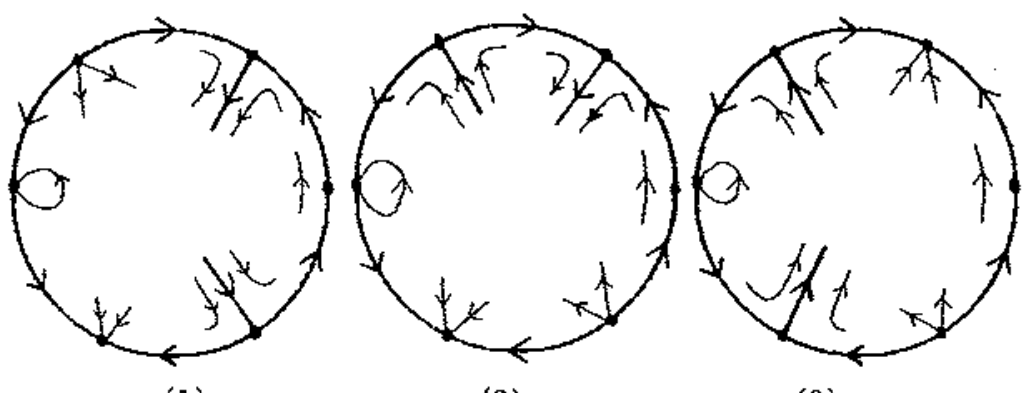

(1)

(2)

(3)

Figure 27. 


\subsection{Realizations.}

In this subsection we shall give chordal cubic systems of type (II) that realize all the possible topological phase portraits of Subsection 5.2.

Phase portraits 10, 15, 19, 20 and 24.

From the proof of Propositions 5.2.1 and 5.2.2, these phase portraits are such that the behaviour at infinity determines completely the phase portrait.

Proposition 5.3.1. System

$$
\begin{aligned}
& \dot{x}=x^{2} y+y^{3}, \\
& \dot{y}=1+x^{2}+2 x y^{2},
\end{aligned}
$$

is a CCS(II) which realizes phase portrait 10.

Proof: By easy computations it follows that system (5.3.1) is a CCS(II). From Appendices A of [6] and I, and Lemma 5.1.2 it follows that: $(1,0)$ is an attractor node, $(-1,0)$ is a repellor node and $(0,0)$ is a critical point of type NE. By Proposition 3.2.1, $(0,0)$ is a saddle. So the behaviour at infinity of system (5.3.1) is shown in Figure 23 and we are done.

Proposition 5.3.2. System

$$
\begin{aligned}
& \dot{x}=(x+y)^{3}, \\
& \dot{y}=1+y(x+y)(x+3 y),
\end{aligned}
$$

is a CCS(II) which realizes phase portrait 15.

Proof: Clearly, system (5.3.2) is a CCS(II). From Lemma 5.1.2 and Appendices $A$ of $[6]$ and I (see also Lemma 5.1.1), we have that $(1,0)$ is an attractor node, $(0,0)$ is a saddle-node of type DE2, and $(-1,0)$ is a critical point of type DE. As in the proof of Lemma 4.3.2, $(-1,0)$ is a saddle-node of type DE1. Then, by Proposition 3.1.1 the behaviour at infinity of system (5.3.2) is like in Figure 25.(1). So the proposition follows.

Proposition 5.3.3. System

$$
\begin{aligned}
& \dot{x}=(1+x+y)\left(1+y^{2}\right), \\
& \dot{y}=(x+y)\left(2+x+y^{2}\right),
\end{aligned}
$$

is a CCS(II) which realizes phase portrait 19.

Proof: By easy computations it follows that system (5.3.3) is a CCS(II). From Appendices $A$ of $[6]$ and $I$ and Lemma $5.1 .2,(1,0)$ is an attractor node, $(0,0)$ and $(-1,0)$ are critical points of type $N E$ and $D E$, respectively. Then, since $l_{1}=0, l_{2}=1, p_{1}=0, p_{2}=1 / 2$ and $p_{3}=1$, by Theorem DE and Proposition 3.2.4, the behaviour at infinity of system (5.3.3) is shown in Figure 26.(1) and so the proposition follows. 
Proposition 5.3.4. System

$$
\begin{aligned}
& \dot{x}=x y^{2}+y^{3}, \\
& \dot{y}=-1-x^{2}+x y^{2}+y^{3},
\end{aligned}
$$

is a CCS(II) which realizes phase portrait 20 .

Proof: It is clear that system (5.3.4) is a CCS(II). As in the proof of Proposition $5.3 .3,(1,0)$ is an attractor node, $(0,0)$ and $(-1,0)$ are critical points of type $\mathrm{NE}$ and $\mathrm{DE}$, respectively. Since $l_{1}=0, l_{2}=-1, p_{1}=0, p_{2}=1 / 2$ and $p_{3}=1$, by Theorem DE and Proposition 3.2.4, the behaviour at infinity of system (5.3.4) is given in Figure 26.(2). Hence, the proposition follows.

\section{Proposition 5.3.5. System}

$$
\begin{aligned}
& \dot{x}=(1+x+y)\left(1-2 x-2 y+y^{2}\right), \\
& \dot{y}=(x+y)\left(1-x-y+y^{2}\right),
\end{aligned}
$$

is a CCS(II) which realizes phase portrait 24 .

Proof: By direct computations it follows that system (5.3.5) is a $\mathrm{CCS}(\mathrm{II})$. As in the proof of Proposition 5.3.3, $(1,0)$ is an attractor node, $(0,0)$ and $(-1,0)$ are critical points of type $N E$ and $D E$, respectively. Since $l_{1}=-2, l_{2}=-1$, $p_{1}=0, p_{2}=1 / 2$ and $p_{3}=1$, by Theorem $\mathrm{DE}$ and Proposition 3.2.4, the proposition follows as in the proof of Proposition 5.3.3.

Phase portraits 16,17 and 18 .

From the proof of Proposition 5.2.2, these phase portraits have the same behaviour at infinity. We consider the family of $C S$

(5.3.6.a)

$$
\dot{x}=(-1+x+y)\left(-5 / 2+(3 / 2) x+(3 / 2) y-2 x^{2}+x y+y^{2}\right),
$$

$$
\dot{y}=(x+y)\left(9+a x+(2-a) y-2 x y+2 y^{2}\right),
$$

with $a \in[-4,8]$. All the systmes of this family are of type (II).

Lemma 5.3.6. If $a \in[-4,8]$ then system (5.3.6.a) is a CCS (II).

Proof: We need to analyse the three systems:

$$
\begin{aligned}
& -1+x+y=0,9+a x+(2-a) y-2 x y+2 y^{2}=0 \\
& x+y=0,-5 / 2+(3 / 2) x+(3 / 2) y-2 x^{2}+x y+y^{2}=0 \\
& 9+a x+(2-a) y-2 x y+2 y^{2}=0 \\
& -5 / 2+(3 / 2) x+(3 / 2) y-2 x^{2}+x y+y^{2}=0
\end{aligned}
$$


In the first one, substituting the first equation into the second one, we have that it has no real solutions if $a \in(-4.3,8.3)$. Similarly, the second system has no real solutions.

In the last system, after some calculations we obtain that it has no real solutions if $a \in(-6.5,8.5)$. Hence the lemma follows.

Now we study the infinite critical points of this family of systems.

Lemma 5.3.7.

(a) System $U_{1}$ associated to system $(5.3 .6 . a)$ is such that, $(1,0)$ is an attractor node, $(0,0)$ is a saddle-node of type DE2 and $(-1,0)$ is a saddle-node of type DE1.

(b) The behaviour at infinity of systems (5.3.6.a) is shown in Figure 25. (2).

Proof: By Lemma 5.1.1 and Appendices A of $[6]$ and $I,(1,0),(0,0)$ and $(-1,0)$ are the critical points of system $U_{1}$, associated to system $(5.3 .6 . \mathrm{a})$, on $z=0$, all them of type DE. By Theorem DE and Proposition 3.1.1, $(0,0)$ and $(-1,0)$ are saddle-nodes and their separatrices are situated as it is indicated in Figure 25.(2). As in the proof of Lemma 4.3.2, we have that $(1,0)$ is an attractor node. So we have proved the lemma.

\section{Proposition 5.3.8.}

(a) System (5.3.6.a) with $a=-3$ realizes phase portrait 16 .

(b) System (5.3.6.a) with $a=7$ realizes phase portrait 18.

(c) System (5.9.6.a) for some $a \in(-3,7)$ realizes phase portrait 17 .

Proof: From Figure 25.(2), to realize phase portrait 16 it is enough to prove that the straight line $f(x, y)=y+x+1=0$ is a solution of system (5.3.6.a) for some $a$. More precisely, we impose that $\operatorname{grad} f(x, y) \cdot(\dot{x}, \dot{y})=\dot{x}+\dot{y}=0$ on $f(x, y)=0$. Since $\dot{x}+\dot{y}=-(a+3)(1+2 x)$ on $f(x, y)=0$, system (5.3.6.a) with $a=-3$ has $f(x, y)=0$ as a solution. Then, from Lemmas 5.3.6 and 5.3.7 it follows (a).

To realize phase portrait 18 we look at the vector field of system (5.3.6.a) on the straight line $g(x, y)=y-3 / 2=0$. We have that $\operatorname{grad} g(x, y) \cdot(\dot{x}, \dot{y})=\dot{y}=$ $(3 / 2+x)[(3 / 2)(11-a)+(a-3) x]$ on $g(x, y)=0$. Since $\dot{y}=(3+2 x)^{2}$ if $a=7$, from Figure 25.(2) and Lemmas 5.3.6 and 5.3.7, (b) is proved.

Statement (c) follows considering similar arguments to those used in the proof of Proposition 4.3.3.(c).

Phase portraits 21, 22 and 23.

From the proof of Proposition 5.2.2, these phase portraits have the same behaviour at infinity. We consider the family of $C S$

$$
\begin{aligned}
& \dot{x}=1+2 x^{2}+x y^{2}+y^{3}, \\
& \dot{y}=d+x^{2}-3 y^{2}+x y^{2}+y^{3},
\end{aligned}
$$

with $d<1$. All the systems of this family are of type (II). 
Lemma 5.3.9. If $d<1$ then system (5.9.7.d) is a $\operatorname{CCS}(I I)$.

Proof: The critical points of system (5.3.7.d) are solution of the system

$$
\begin{aligned}
& A(x, y)+P_{3}(x, y)=0, \\
& B(x, y)+P_{3}(x, y)=0,
\end{aligned}
$$

or equivalently

$$
\begin{aligned}
& A(x, y)+P_{3}(x, y)=0, \\
& B(x, y)-A(x, y)=0,
\end{aligned}
$$

where $P_{3}(x, y)=x y^{2}+y^{3}$. Since $B(x, y)-A(x, y)=(d-1)-x^{2}-3 y^{2}$, the lemma follows.

Now we study the infinite critical points of this family of systems.

\section{Lemma 5.3.10.}

(a) System $U_{1}$ associated to system $(5.3 .7 . d)$ is such that $(1,0)$ is an attractor node, $(0,0)$ is a saddle-node of type $N E$ and $(-1,0)$ is a saddle-node of type DE1.

(b) The behaviour at infinity of systems (5.3.7.d) is shown in Figure 26.(3).

Proof: By Lemma 5.1.1, $(1,0),(0,0)$ and $(-1,0)$ are the critical points of system $U_{1}$ on $z=0$. From Appendices $A$ of $[6]$ and $I(1,0)$ is an attractor node, $(0,0)$ is of type NE and $(-1,0)$ is of type DE. Since $p_{1}=0, p_{2}=1 / 2$, $p_{3}=1, l_{1}=2$ and $l_{2}=1$, by Theorem DE and Proposition $3.2 .4,(0,0)$ and $(-1,0)$ are saddle-nodes and their separatrices are situated as it is shown in Figure 26.(3).

\section{Proposition 5.3.11.}

(a) System (5.3.7.d) with $d=-1$ realizes phase portrait 21 .

(b) System (5.3.7.d) with $d=1 / 2$ realizes phase portrait 23 .

(c) System (5.9.7.d) for some $d \in(-1,1 / 2)$ realizes phase portrait 22.

Proof: From Figure 26.(3) to realize phase portrait 21 it is enough to prove that the straight line $f(x, y)=x+y=0$ is a solution of system (5.3.7.d) for some $d$. More precisely, we impose that $\operatorname{grad} f(x, y) \cdot(\dot{x}, \dot{y})=\dot{x}+\dot{y}=0$ on $f(x, y)=0$. Since $\dot{x}+\dot{y}=1+\dot{d}$ on $f(x, y)=0$; system (5.3.7.d) with $d=-1$ has $f(x, y)=0$ as a solution. Then, fron Lemmas 5.3 .9 and 5.3 .10 it follows (a).

To realize phase portrait 23 we look at the vector field of system (5.3.7.d) on the straight line $g(x, y)=y=0$. Since $\operatorname{grad} g(x, y) \cdot(\dot{x}, \dot{y})=d+x^{2}$ on $g(x, y)=0$, if $d=1 / 2 g(x, y)=0$ is a curve without contact points for system (5.3.7.d). Then, from Figure 26.(3) and Lemmas 5.3.9 and 5.3.10 (b) is proved. 
Statement (c) follows as in the proof of Proposition 4.3.3.(c).

Phase portraits 11,25 and 26.

From the proof of Propositions 5.2.1 and 5.2.2, these phase portraits are such that the behaviour at infinity determines the phase portrait.

Proposition 5.3.12. System

$$
\begin{aligned}
& \dot{x}=(-1+y)\left(-20-x^{2}+x y+y^{2}\right), \\
& \dot{y}=x^{2}+y^{3},
\end{aligned}
$$

is a CCS(II) which realizes phase portrait 11 .

Proof: Looking at the flow on the straight line $y-1=0$, on the hyperbola $y^{2}+x y-x^{2}=20$ and on the cubic $x^{2}+y^{3}=0$, it follows that system (5.3.8) is chordal.

From Lemma 5.1.2 and Appendices A of [6] and I (see also Lemma 5.1.1) we have that $(1,0)$ is an attractor node, $(0,0)$ is a critical point of type NE and $(-1,0)$ is a saddle. Then, since $p_{1}=0, p_{2}=-1 / 2, p_{3}=I$ and $l_{2}=1$, from Proposition $3.2 .1,(0,0)$ is the union of a hyperbolic and elliptic sector. From Proposition 3.2.3 the behaviour at infinity of system (5.3.8) is shown in Figure 24.(1). So the proposition follows.

Proposition 5.3.13. System

$$
\begin{aligned}
& \dot{x}=-2 y-x^{2} y+y^{3}, \\
& \dot{y}=1+x^{2}-4 y^{2},
\end{aligned}
$$

is a CCS(II) which realizes phase portrait 25.

Proof: By easy computations it follows that system (5.3.9) is a CCS(II). As in the proof of Proposition 5.3.12, $(1,0)$ and $(-1,0)$ are critical points of type $\mathrm{DE}$ and $(0,0)$ is a critical point of type NE. Since $p_{1}=0, p_{2}=-1 / 2, p_{3}=0$ and $l_{2}=1$, from Theorem DE, Lemma 5.1.2 and Propositions 3.2.1 and 3.2.3, $(1,0)$ and $(-1,0)$ are saddle-nodes of type DE1 and $(0,0)$ is the union of a hyperbolic and elliptic sector. Therefore the behaviour at infinity of system (5.3.9) is shown in Figure 27.(1). Hence the proposition follows.

Proposition 5.3.14. System

$$
\begin{aligned}
& \dot{x}=x+y-x^{2} y+y^{3}, \\
& \dot{y}=-1+x^{2}+(3 / 2) x y-y^{2},
\end{aligned}
$$

is a CCS(II) which realizes phase portrait 26 .

Proof: Studying the flow on the straight line $x+y=0$ and on the hyperbolas $y^{2}-x y=-1$ and $x^{2}+(3 / 2) x y-y^{2}=1$, it follows that system (5.3.10) is chordal. Now, the proposition follows as in the proof of Proposition 5.3.13. 
Phase portraits 12,13 and 14.

From the proof of Proposition 5.2.1, these phase portraits have the same behaviour at infinity. We consider the family of $C S$

$$
\begin{aligned}
& \dot{x}=2 b-x+(b-4) y+x^{2} / 2-x^{2} y+x y^{2}+y^{3}, \\
& \dot{y}=(1+y)\left(1+b y+y^{2}\right),
\end{aligned}
$$

with $b \in[-2,2]$. All the systems of this family are of type (II).

Lemma 5.2.15. If $b \in[-2,2]$ then system $(5.9 .11 . b)$ is a chordal cubic system.

Proof: First we consider $b \in[-2,2]$. Then $\dot{y}=0$ if and only if $y=-1$. Moreover, $\dot{x}=b+3+(3 / 2) x^{2}$ on $y=-1$.

Now, we assume that $b=-2$. Clearly $\dot{y}=0$ if and only if $y=1$ or $y=-1$. Since $\dot{x}=-9-x^{2} / 2$ on $y=1$ and $\dot{x}=1+(3 / 2) x^{2}$ on $y=-1$, the lemma follows.

Now we study the infinite critical points of this family of systems.

\section{Lemma 5.3.16.}

(a) System $U_{1}$ associated to system $(5.3 .11 . b)$ is such that $(1,0)$ is an attractor node, $(0,0)$ is the union of a hyperbolic and elliptic sector of type $Z$ and $(-1,0)$ is a saddle. 24.(2).

(b) The behaviour at infinity of systems (5.9.11.b) is shown in Figure

Proof: By Lemma 5.1.1, $(1,0),(0,0)$ and $(-1,0)$ are the critical points of system $U_{1}$ on $z=0$. From Appendices $A$ of $[6]$ and $I(1,0)$ is an attractor node, $(0,0)$ is a critical point of type $Z$ and $(-1,0)$ is a saddle. To analyse the behaviour at $(0,0)$ we consider the blow up $y=y, z=w y$. Therefore, system $U_{1}$ is equivalent (after omitting a common factor $y^{2}$ ) to

$$
\begin{aligned}
& \dot{y}=1-w / 2+y Y(y, w), \\
& \dot{w}=-w(1+w)\left(1+b w+w^{2}\right),
\end{aligned}
$$

where $Y$ is a polynomial in $y$ and $w$. Looking at the vector field of this system on the $w$-axis and unmaking the change of variables we obtain the Figure 28. Hence, the lemma follows. 
Remark 5.3.17. Note that system (5.3.11.b) is not a CCS(II) since the origin of system $U_{1}$ has linear part identically zero.

\section{Proposition 5.3.18.}

(a) System (5.8.11.b) with $b=-2$ realizes phase portrait 14 .

(b) System $(5.9 .11 . b)$ with $b \in(1,2]$ realizes phase portrait 12 .

(c) System $(5.3 .11 . b)$ for some $b \in(-2,1)$ realizes phase portrait 13 .

Proof: It is easy to prove that the straight line $y+1=0$ is a solution of the system (5.3.11.b) for any $b \in[-2,2]$. Also the straight tine $y-1=0$ is a solution of system (5.3.11.b) when $b=-2$. Looking at the vector field of this system on the $y$-axis we have

$$
\begin{aligned}
& \dot{x}=(2+y)\left(b-2 y+y^{2}\right), \\
& \dot{y}=(1+y)\left(1+b y+y^{2}\right) .
\end{aligned}
$$

on $x=0$. From Figure 29 and Lemmas 5.3.15 and 5.3.16, (a) and (b) follows.

Statement (c) follows as in the proof of Proposition 4.3.3.(c).
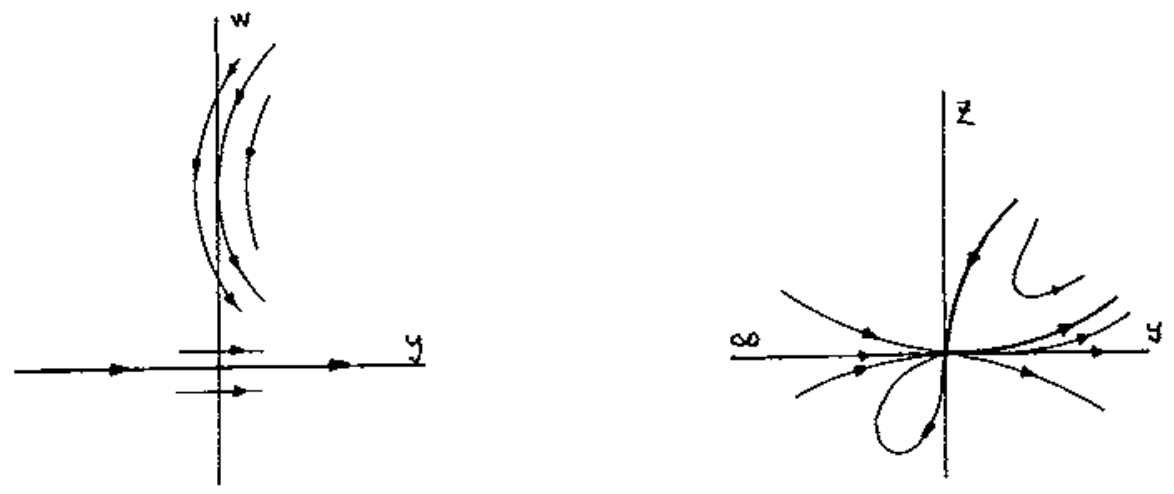

Figure 28.

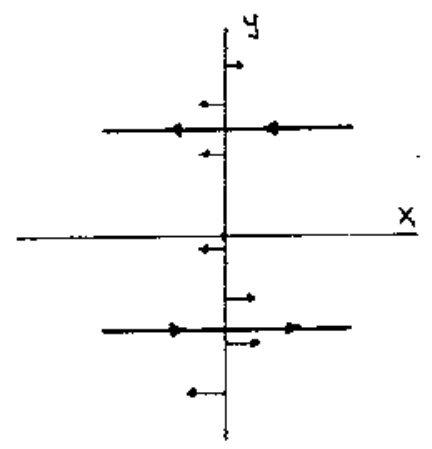

$b=-2$

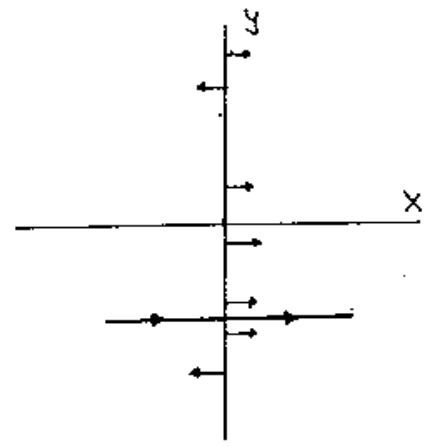

$b \in(-2,1)$ 


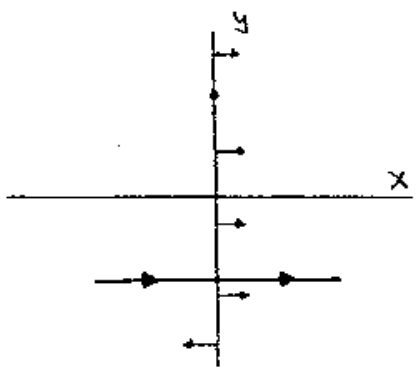

$$
b=1
$$

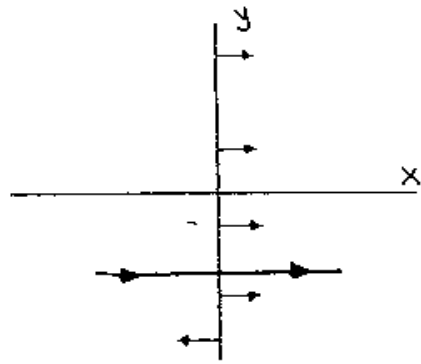

$b \in(1,2]$

Figure 29.

Phase portraits 27 and 28.

From the proof of Proposition 5.2.2, these phase portraits have the same behaviour at infinity. We consider the family of $C S$

$$
\dot{x}=1+a x+y-x^{2}+2 x y+y^{2}-x^{2} y+y^{3},
$$

$$
\dot{y}=1+x^{2}+y^{2} .
$$

It is clear that system (5.3.12.a) is a CCS(II) for all $a \in R$. In the next lemma we study their infinite critical points.

\section{Lemma 5.3.19.}

(a) System $U_{1}$ associated to systems $(5.9 .12 . a)$ is such that $(1,0)$ and $(-1,0)$ are saddle-nodes of type DE1 and $(0,0)$ is the union of a hyperbolic and elliptic sector. 27.(3).

(b) The behaviour at infinity of systems (5.3.12.a) is shown in Figure

The proof follows as in the proof of Propositions 5.3.13 and 5.3.14.

\section{Proposition 5.3.20.}

(a) System (5.8.12.a) with $a=0$ realizes phase portrait 27.

(b) System (5.3.12.a) with $a \neq 0$ realizes phase portrait 28 .

Proof: We look at the vector field of system (5.3.12.a) on the hyperbola $f(x, y)=y^{2}-x^{2}+1=0$. Since grad $f(x, y) \cdot(\dot{x}, \dot{y})=-a x^{2}$ on $f(x, y)=0$, from Figure 27.(3) and Lemma 5.3.19, the proposition follows. 


\section{System (III)}

In this section we determine all the possible phase portraits of a CCS(III). Also we realize these phase portraits.

\subsection{Critical points.}

Now we study the critical points at infinity of system (III) (see Remark 4.1.1). An easy computation (see Appendix $A$ of [6]) allows to prove the next result.

Lemma 6.1.1. System $U_{1}$ associated to system (III) has two critical points on $z=0:(1,0)$ and $(-1,0)$.

The next lemma follows from Apendices $A$ of $[6]$ and I.

\section{Lemma 6.1.2.}

(a) The critical point $(1,0)$ of system $U_{1}$ associated to system (III) is of type $E$ if $p_{1}+p_{2}+p_{3} \neq \mu$, and of type $D E$ if $p_{1}+p_{2}+p_{3}=\mu$.

(b) The critical point $(-1,0)$ of system $U_{1}$ associated to system (III) is of type $E$ if $-p_{1}+p_{2}-p_{3} \neq \mu$, and of type $D E$ if $-p_{1}+p_{2}-p_{3}=\mu$.

In both cases, if the DE critical point is a saddle-node, then it is of type DE1.

\subsection{Topological phase portraits and realizations.}

In this subsection we study all the possible topological phase portraits of a CCS(III) and we realize them.

\section{Proposition 6.2.1.}

(a) The phase portrait of a CCS(II) is homeomorphic (except, perhaps the orientation) to phase portrait 29 .

(b) System

$$
\begin{aligned}
& \dot{x}=\mu x^{3}-\mu y^{3}, \\
& \dot{y}=1-\mu x^{3}+\mu x^{2} y,
\end{aligned}
$$

with $\mu>0$, is a CCS(III) which realizes phase portrait 29 .

Proof: From Lemma 6.1.2 and Theorems E, DE and Poincaré-Hopf Theorem, the critical points at infinity are (see Lemma 6.1.1) one node and one saddle-node of type DE1.

By using symmetries, rotations or changes of sign in the variable $t$ (if it is necessary) we obtain that any CCS(III) has a behaviour at infinity as in Figure 30. This figure determines phase portrait 29. Hence (a) follows.

By easy computations we have that system (6.2.1) is a CCS(III). From Appendices $A$ of $[6]$ and $I(1,0)$ is a critical point of type $D E$ and $(-1,0)$ 
is an attractor node. As in the proof of Proposition 4.3 .2 it follows that $(1,0)$ is a saddle-node of type DE1. Then from (a) it follows (b).

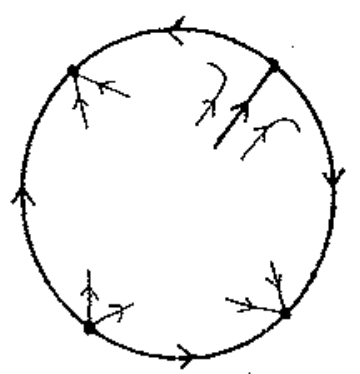

Figure 30 .

\section{System (IV)}

In this section we determine all the possible phase portraits of a CCS(IV). Also we realize these phase portraits.

\subsection{Critical points.}

Now we study the critical points at infinity of system (IV) (see Remark 4.1.1). An easy computation (see Appendix A of [6]) allows to prove the next result.

\section{Lemma 7.1.1.}

(a) System $U_{1}$ essociated to system (IV) has only the critical point $(0,0)$ on $z=0$.

(b) System $U_{2}$ associated to system (IV) has only the critical point $(0,0)$ on $z=0$.

The next two lemmas follow from Appendices $A$ of $[6]$ and $I$, see also Propositions 3.1 .1 and 3.1 .2 .

\section{Lemma 7.1.2.}

(a) The critical point $(0,0)$ of system $U_{1}$ associated to system (IV) is of type $D E$ if $p_{1} \neq 0$, of type $N E$ if $p_{1}=0, l_{2} \neq 0$, and of type $Z$ if $p_{1}=l_{2}=0$.

(b) The critical point $(0,0)$ of system $U_{2}$ associated to system (IV) is of type $D E$ if $p_{3} \neq 0$, of type $N E$ if $p_{3}=0, n_{1} \neq 0$, and of type $Z$ if $p_{3}=n_{1}=0$.

In both cases, if the critical point is a DE saddle-node, it is of type DE2.

Lemma 7.1.3. The vector field on the equator of the Poincaré sphere of system (IV) is shown in Figure 31. 


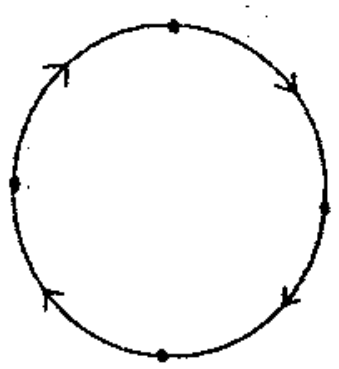

Figure 31.

\subsection{Topological phase portraits.}

In this subsection we study all the possible topological phase portraits of a CCS(IV).

\section{Proposition 7.2.1.}

(a) The critical points at infinity of a CCS(IV) are a saddle-node and the union of a hyperbolic and elliptic sector.

(b) The phase portrait of a CCS(IV) is homeomorphic (except, perhaps the orientation) to one of phase portraits $30,31,32,39,94$ and 35.

Proof: From Lemma 7.1.2 and Theorems DE, NE and Poincaré-Hopf Theorem the two critical points at infinity (see Lemma 7.1.1) have indices 1 and 0 . Again from Theorems DE and NE and Lemma 7.1.3, (a) follows.

Without loss of generality we can suppose that $(0,0)$ of system $U_{1}$ is the union of a hyperbolic and elliptic sector. Therefore from Lemma 7.1.2 and (a) we consider two cases. Case 1 (resp. Case 2): $(0,0)$ of system $U_{2}$ is a saddle-node of type DE2 (resp. of type NE).

In the first case, from Propositions 3.1.2 and 3.2 .3 and by using symmetries, rotations and changes of sign if it is necessary, it follows that any CCS(IV) has a behaviour at infinity as in Figure 32 . In the second case, from Propositions 3.2.3, 3.3.1 and 3.3.4 any CCS(IV) has a behaviour at infinity like in Figure 33.

Figures 32.(1), 33.(1) and 33.(2) determine phase portraits 30,34 and 35; respectively. Figure $32 .(2)$ determines phase portraits 31,32 and 33 . Hence (b) follows. 


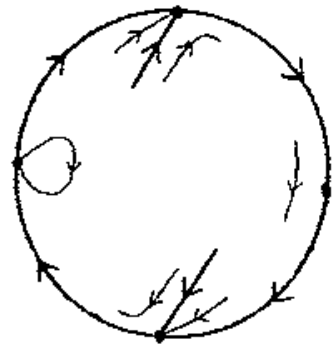

(1)

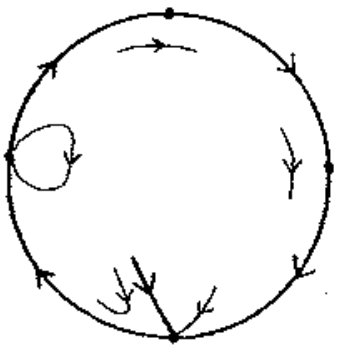

(1)

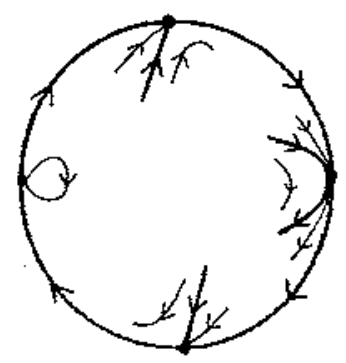

(2)

Figure 32.

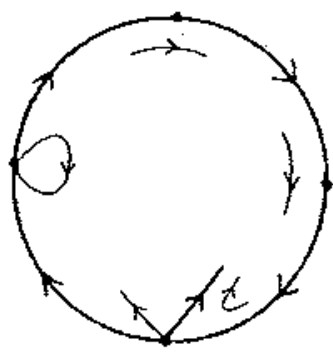

(2)

Figure 33 .

\subsection{Realizations.}

In this subsection we shall give chordal cubic systems of type (IV) that realize all the possible topological phase portraits of Subsection 7.2 .

Phase portraits 30,34 and 35 .

From the proof of Proposition 7.2.1, these phase portraits are such that the behaviour at infinity determines the phase portrait.

Proposition 7.3.1. System

$$
\begin{aligned}
& \dot{x}=-9+6 x^{2} y+x y^{2}, \\
& \dot{y}=x^{2}+y^{3},
\end{aligned}
$$

is a CCS(IV) which realizes phase portrait So.

Proof: From the flow on the cubics $x^{2}+y^{3}=0$ and $x y(6 x+y)=9$ we can prove that system (7.3.1) is chordal. 

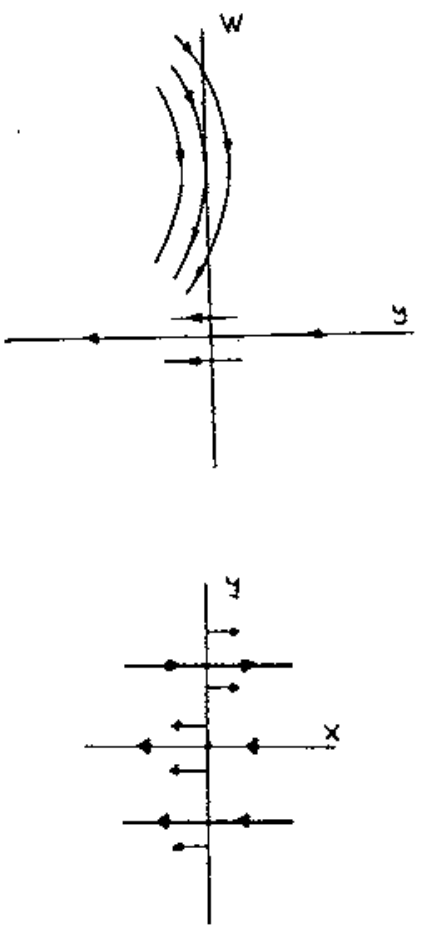

$b=-2$

Figure 34 .

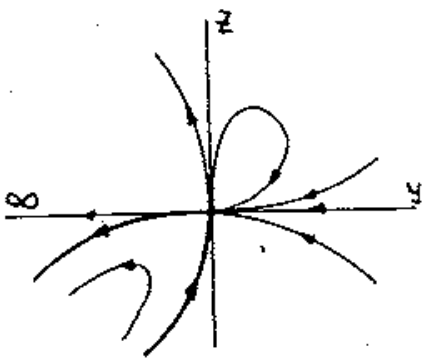

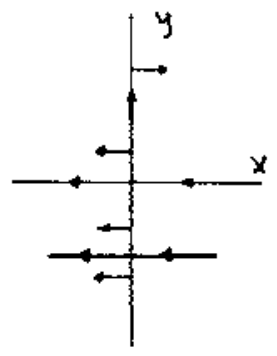

$b \in(-2,2)$

Figure 35 .

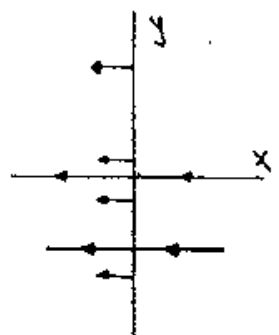

$b=2$

\section{System (V)}

In this section we determine all the possible phase portraits of a $\operatorname{CSS}(\mathrm{V})$ and we realize them.

\subsection{Critical points.}

Now we study the critical points at infinity of system(V) (see Remark 4.1.1). An easy computation (see Appendix A of [6]) allows to prove the next result.

\section{Lemma 8.1.1.}

(a) System $U_{1}$ associated to system (V) has only one critical point on $z=0$, the origin.

(b) System $U_{2}$ associated to system (V) has only one critical point on $z=0$, the origin.

The next two lemmas follow from Appendices A of [6] and $I$. 
Lemma 8.1.2.

(a) The critical point $(0,0)$ of system $U_{1}$ associated to system (V) is of type $E$ if $p_{1} \neq-2$, and of type $D E$ if $p_{1}=-2$. Moreover, if the critical point is a $D E$ saddle-node, it is of type $D E 1$.

(b) The critical point $(0,0)$ of system $U_{2}$ associated to system (V) is of type $D E$ if $p_{3} \neq 0$, of type $N E$ if $p_{3}=0, n_{1} \neq 0$, and of type $Z$ if $p_{3}=n_{1}=0$.

Lemma 8.1.3. The vector field on the equator of the Poincare sphere of system (V) is shown in Figure 36.

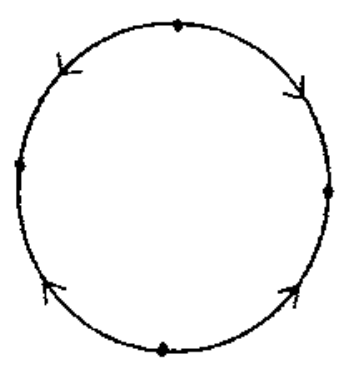

Figure 36 .

\subsection{Topological phase portraits.}

In this subsection we study all the possible topological phase portraits of a $\operatorname{CCS}(\mathrm{V})$.

\section{Proposition 8.2.1.} node.

(a) The critical points at infinity of a CCS(V) are a saddle-node and a

(b) The phase portrait of a CCS $(V)$ is homeomorphic (except, perhaps the orientation) to one of phase portraits 29 and 96 .

Proof: From Lemma 8.1.2 and Theorems E, DE, NE and Poincaré-Hopf Theorem, the two critical points at infinity (see Lemma 8.1.1) have indices 1 and 0. Again, from Theorems E, DE, and NE and Propositions 3.1.2 and 3.3.1, (a) follows; and furthermore we have two cases. Case 1: the origin of system $U_{1}$ is a node and the origin of system $U_{2}$ is a saddle-node of type $\mathrm{NE}$. Case 2: the origin of system $U_{1}$ is a saddle-node of type $\mathrm{DEI}$ and the origin of system $U_{2}$ is a node.

In the first case, to study the saddle-node of type $\mathrm{NE}$, we must analyse two options (see Proposition 3.3.1).

Option 1: $p_{2} \neq 0$. This option is studied in Proposition 3.3.4, and the behaviour at infinity is shown in Figure 37. 
Option 2: $p_{2}=0$ plus other conditions. From Lemma 8.1.3, only Figures 11.(3) and 11.(4) can occur. Figure 11.(3) is such that the behaviour of the saddle-node is topologically equivalent to a saddle-node of type DE1. Then by a rotation we are in Case 2. Figure 11.(4) gives the same situation that in Option 1.

In the second case we have that the behaviour at infinity is shown in Figure 38.

Figures 37 and 38 determine phase portraits 36 and 29 , respectively. Hence, (b) follows.

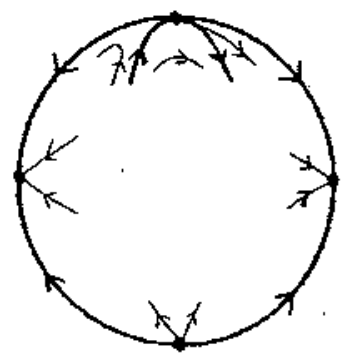

Figure 37 .

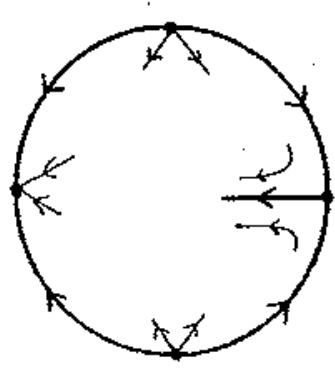

Figure 38 .

\subsection{Realizations.}

In this subsection we shall give a $\mathrm{CCS}(\mathrm{V})$ which realizes phase portrait 36 , because phase portrait 29 is realized in Proposition 6.2.1.

Phase portrait 36.

From the proof of Proposition 8.2 .1 , the behaviour at infinity determines the phase portrait.

Proposition 8.3.1. System

$$
\begin{aligned}
& \dot{x}=1+y^{2}+4 x^{3}+x^{2} y, \\
& \dot{y}=x+x y^{2},
\end{aligned}
$$

is a $\operatorname{CCS}(V)$ which realizes phase portrait 36 .

Proof: Clearly system (8.3.1) is a CCS(V). For this system we have $p_{1}=2$, $p_{2}=1, p_{3}=0, n_{2}=1$. So, from Lemma 8.1.2, Theorem $E$ and Proposition 3.3.4 the behaviour at infinity of system (8.3.1) is shown in Figure 37 and we are done.

\section{System (VI)}

In this section we determine all the possible phase portraits of a CCS(VI). Also we realize these phase portraits. 
9.1. Critical points and topological phase portraits.

Now we study the critical points at infinity of system (VI) (see Remark 4.1.1). From Appendices $A$ of $[6]$ and $I$ it follows the next result.

\section{Lemma 9.1.1.}

(a) System $U_{1}$ associated to system (VI) has only one critical point on $z=0$, the origin.

(b) This critical point is of type $D E$ if $p_{1} \neq 0$, of type $N E$ if $p_{1}=0, l_{2} \neq 0$, and of type $Z$ if $p_{1}=l_{2}=0$.

Now we deduce all the possible topological phase portraits of a CCS(VI).

\section{Proposition 9.1.2.}

(a) The critical point at infinity of a CCS(VI) is the union of a hyperbolic. and elliptic sector.

(b) The phase portrait of a CCS(VI) is homeomorphic (except, perhaps the orientation) to one of phase portraits 37 and 38 .

Proof: From Lemma 9.1.1 and Theorems DE, NE and Poincaré-Hopf Theorem the critical point at infinity has index 1 and it is of type DE or NE. Hence, from Propositions 3.1.1 and 3.2.1, (a) follows.

From Proposition 3.2 .3 and by using rotations, any CCS(VI) has a behaviour at infinity as in Figure 39. Figures 39.(1) and 39.(2) determine phase portraits 37 , and 38 , respectively.

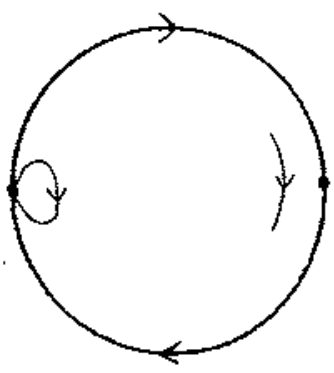

(1)

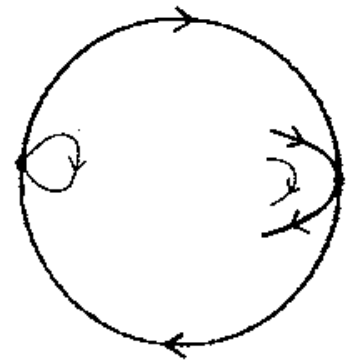

(2)

Figure 39.

\subsection{Realizations.} 38 .

In this subsection we shall give CCS(VI) which realize phase portraits 37 and

Phase portraits 37 and 38.

From the proof of Proposition 9.1.2, these phase portraits are such that the behaviour at infinity determines the phase portrait. 
Proposition 9.2.1. Systems

$$
\begin{aligned}
& \dot{x}=6 x^{2} y+y^{3}, \\
& \dot{y}=1+x^{2},
\end{aligned}
$$

and

$$
\begin{aligned}
& \dot{x}=13 x^{2} y+y^{3}, \\
& \dot{y}=1+x^{2}+7 x y^{2},
\end{aligned}
$$

are CCS(VI) which realize phase portraits 97 and 98 , respectively.

The proof follows easily from Proposition 3.2.3 and Figure 39.

\section{System (VII)}

In this section we determine all the possible phase portraits of a CCS(VII).

\subsection{Critical points and topological phase portraits.}

From Appendices $A$ of $[6]$ and $I$ it follows the next result.

\section{Lemma 10.1.1.}

(a) System $U_{2}$ associated to system (VII) has only one critical point on $z=0$, the origin.

(b) This critical point is of type $D E$ if $p_{3} \neq 0$, of type $N E$ if $p_{3}=0, n_{1} \neq 0$, and of type $Z$ if $p_{3}=n_{1}=0$.

The following proposition gives us the possible topological phase portraits of a CCS(VII).

\section{Proposition 10.1.2.}

(a) The critical point at infinity of a CCS(VII) is the union of a hyperbolic and elliptic sector.

(b) The phase porirait of a CCS(VII) is homeomorphic (except, perhaps the orientation) to one of phase portraits 37 and 98 .

Proof: From Propositions 3.1.2 and 3.3.1, and by using similar arguments to the proof of Proposition 9.1.2, (a) follows.

From the proof of Proposition 3.3.1 we have two options for the critical point $(0,0)$ of system $U_{2}$ associated to system (VII).

Option 1: $p_{2}<0$. This option is studied in Proposition 3.3.3 and the behaviour at infinity is shown in Figure 39.(2).

Option 2: $p_{2}=p_{1}=0$. To analyse the local behaviour at $(0,0)$ we consider the changes of variables $x=x, z=x w_{1} ; x=x, w_{1}=x w ; x=x, w=x u$ and 
$x=x, u=x v$. Therefore, system $U_{2}$ is equivalent (after omitting a common factor $x^{3}$ ) to

$$
\begin{aligned}
& \dot{x}=x+n_{1} x v+x^{2} X(x, v), \\
& \dot{v}=-3 v-4 n_{1} v^{2}+x v V(x, v),
\end{aligned}
$$

where $X$ and $V$ are polynomials in $x$ and $v$ of degree at least one. This system has exactly two critical points on the $v$-axis, they are a saddle at $(0,0)$ and a repellor node at $\left(0,-3 /\left(4 n_{1}\right)\right)$. So, from Figure 40 (where we suppose $n_{1}>0$ ), the behaviour at infinity is shown in Figure 39.(1). Then from the proof of Proposition 9.1.2, the proposition follows.

Remember that, phase portraits 37 and 38 are realized in Proposition 9.2.1.
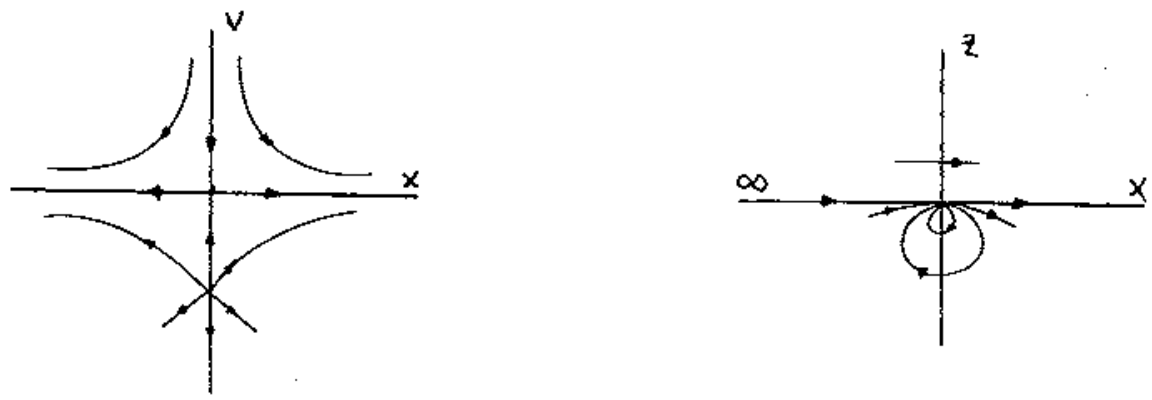

Figure 40.

\section{Systems (VIII), (IX) and (X)}

By using Appendix A of [6] and Remark 4.1.1, systems (VIII) and (IX) has no infinite critical points. Then, from Poincaré-Hopf Theorem, these systems cannot be a CCS. Furthermore, system $(X)$ cannot be a CCS because all the points on the equator of the Poincare sphere are critical points. Hence, the Theorem of Section 1 it is proved.

\section{Appendix I}

This appendix contains the theorems which we use in the study concerning the local behaviour near a critical point of type $\mathrm{E}, \mathrm{DE}$ or NE. Also it contains other results.

Theorem E. (see [2]). Let $(0,0)$ be an isolated critical point of the vector field $X(x, y)=(a x+b y+F(x, y), c x+d y+G(x, y))$, where $F$ and $G$ are analytic in a neighbourhood of the origin and have expansions that begin with 
second degree terms in $x$ and $y$. We say that $(0,0)$ is a nondegenerate critical point if $a d-b c \neq 0$. Let $\mu_{1}$ and $\mu_{2}$ be the eigenvalues of $D X(0,0)$. Then the following hold.

(a) If $\mu_{1}, \mu_{2}$ are real and $\mu_{1} \mu_{2}<0$, then $(0,0)$ is a saddle (Figure B.1.(1)) whose separatrices tend to $(0,0)$ in the directions given by the eigenvectors associated with $\mu_{1}$ and $\mu_{2}$.

(b) If $\mu_{1}, \mu_{2}$ are real and $\mu_{1} \mu_{2}>0$, then $(0,0)$ is a node (Figure B.1.(2)). If $\mu_{1}>0$ (resp. $\left.<0\right)$ then it is a source (resp. sink).

(c) If $\mu_{1}=\alpha+\beta i$ and $\mu_{2}=\alpha-\beta i$ with $\alpha, \beta \neq 0$, then $(0,0)$ is a focus (Figure B.1.(3)). If $\alpha>0$ (resp. <0) then it is repellor (resp. attractor).

(d) If $\mu_{1}=\beta i$ and $\mu_{2}=-\beta i$, then $(0,0)$ is a linear center, topologically a focus or a center (Figute B.1.(4)).

The correponding indices are $-1,+1,+1,+1$.

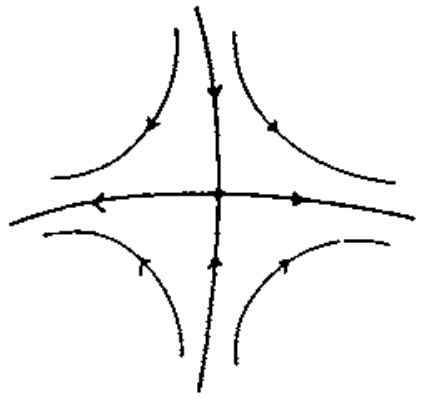

(1)

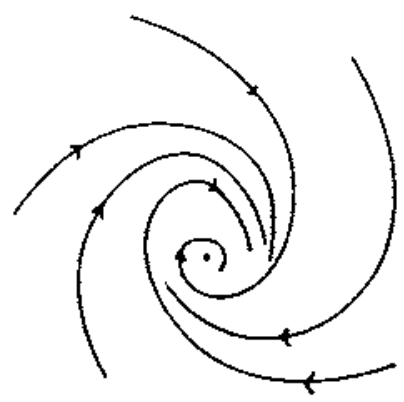

(3)

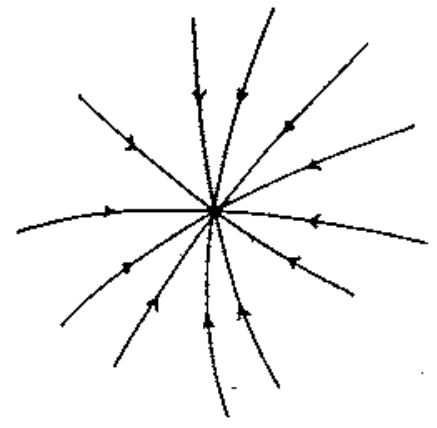

(2)

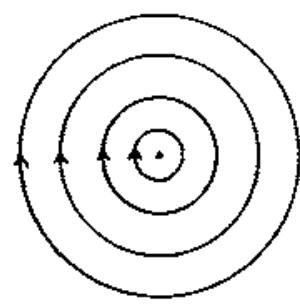

(4)

Figure B.1. The local behaviour near a critical point of type $\mathrm{E}$ (we can reverse the orientation of the orbits). 


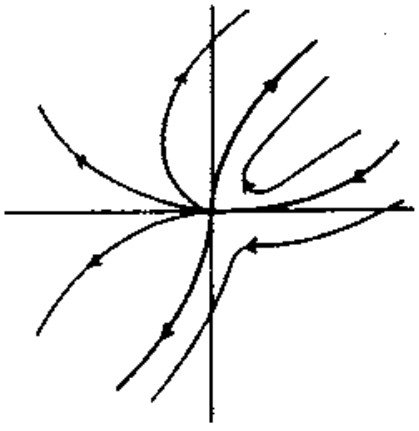

(1)

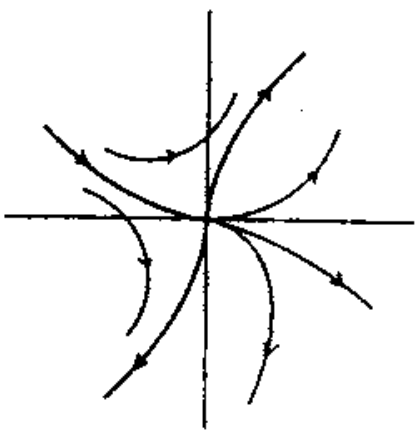

(2)

Figure B.2. The saddle-nodes of type DE (we can reverse the orientation of the orbits).

Theorem DE. (see Theorem 65 of $[2]$ ). Let $(0,0)$ be an isolated critical point of the system

$$
\begin{aligned}
& \dot{x}=X(x, y), \\
& \dot{y}=y+Y(x, y),
\end{aligned}
$$

where $X$ and $Y$ are analytic in the neighbourhood of the origin and have expansions that begin with second degree terms in $x$ and $y$. Let $y=f(x)$ be the solution of the equation $y+Y(x, y)=0$ in a neighbourhood of $(0,0)$, and assume that the series expansion of the function $g(x)=X(x, f(x))$ has the form $g(x)=a_{m} x^{m}+\ldots$, where $m \geq 2, a_{m} \neq 0$.

(a) If $m$ is odd and $a_{m}>0$, then $(0,0)$ is a topological node.

(b) If $m$ is odd and $a_{m}<0$, then $(0,0)$ is a topological saddle, two of whose separatrices tend to $(0,0)$ in the directions 0 and $\pi$, the other two in the directions $\pi / 2$ and $3 \pi / 2$.

(c) If $m$ is even then $(0,0)$ is a saddle-node, i.e. a critical point whose neighbourhood is the union of one parabolic and two hyperbolic sectors, two of whose separatrices tend to $(0,0)$ in the directions $\pi / 2$ and $3 \pi / 2$ and the other in the direction 0 or $\pi$ according to $a_{m}<0$ (Figure B.2.(1)) or $a_{m}>0$ (Figure B.2.(2)).

The corresponding indices are $+1,-1,0 ;$ so they may serve to distinguish the three types.

From Theorems $\mathrm{E}$ and $\mathrm{DE}$, the stable and unstable separatrices of a saddle $p$ of type $\mathrm{E}$ or $\mathrm{DE}$ form an angle into the point $p$. So, the infinity separates the hyperbolic sectors like in Figure B.3. From the Theorem DE the infinity separates a saddle-node $p$ like in Figure B.4. We note that the linear part, 
$D X(p)$, will be either

$$
\left[\begin{array}{cc}
\neq 0 & * \\
0 & 0
\end{array}\right] \text {, or }\left[\begin{array}{cc}
0 & * \\
0 & \neq 0
\end{array}\right] \text { according to } p \text { is of type } D E 1
$$

or $\mathrm{DE} 2$, respectively.

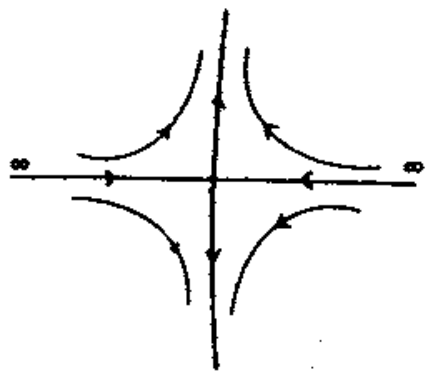

Figure B.3. A saddle of type E or DE on the equator of $S^{2}$ (we can reverse the orientation of the orbits).
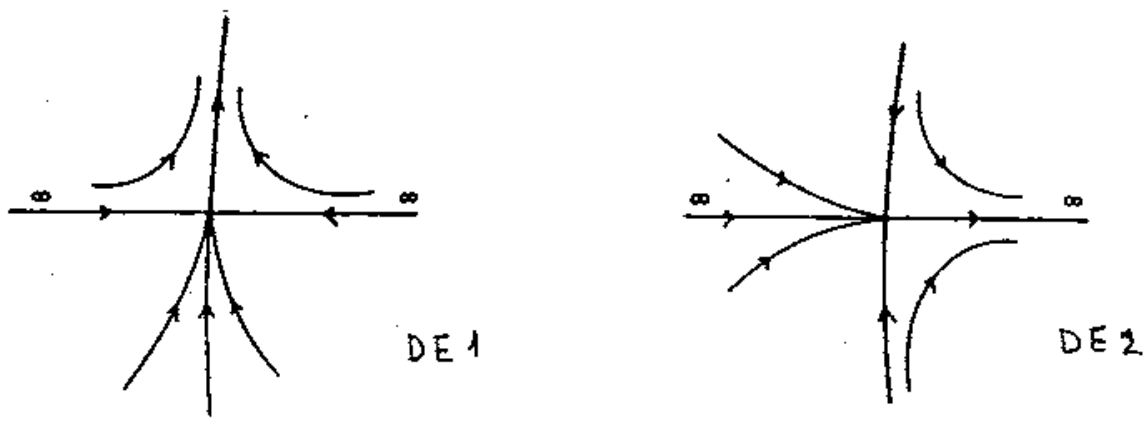

Figure B.4. The saddle-nodes of type DE1 or DE2 of $p(X)$ on the equator of $S^{2}$ (we can reverse the orientation of the orbits).

Theorem NE. (see / $1 /)$. Let $(0,0)$ be an isolated critical point of the system

$$
\begin{aligned}
& \dot{x}=y+X(x, y), \\
& \dot{y}=Y(x, y),
\end{aligned}
$$

where $X$ and $Y$ are analytic in a neighbourhood of the origin and have expansions that begin with second degree terms in $x$ and $y$. Let $y=F(x)=$ $a_{2} x^{2}+a_{3} x^{3}+\ldots$ be $a$ solution of the equation $y+X(x, y)=0$ in the neighbourhood of $(0,0)$, and assume that we have the series expansions for the functions $f(x)=Y(x, F(x))=a x^{\alpha}(1+\ldots)$ and $\Phi(x)=(\partial X / \partial x+\partial Y / \partial y)(x, F(x))=$ $b x^{\beta}(1+\ldots)$ where $a \neq 0, \alpha \geq 2$ and $\beta \geq 1$. 
(a) If $\alpha$ is even, and

(a.1) $\alpha>2 \beta+1$, then the origin is a saddle-node (index 0), see Figure B.5.(1);

(a.2) either $\alpha<2 \beta+1$ or $\Phi(x) \equiv 0$, then the origin is a critical point whose neighbourhood is the union of two hyperbolic sectors (index 0 ), see Figure B.5.(2).

(b) If $\alpha$ is odd and $a>0$, then the origin is a saddle (index-1), see Figure B.5.(3).

(c) If $\alpha$ is odd, $a<0$, and

(c.1) either $\alpha>2 \beta+1$ and $\beta$ even; or $\alpha=2 \beta+1, \beta$ even and $b^{2}+4 a(\beta+$ 1) $\geq 0$, then the origin is a node (index +1 ), see Figure B.5.(4). The node is stable if $b<0$, or unstable if $b>0$;

(c.2) either $\alpha>2 \beta+1$ and $\beta$ odd; or $\alpha=2 \beta+1, \beta$ odd and $b^{2}+4 a(\beta+1) \geq$ 0 , then the origin is the union of a hyperbolic and elliptic sector (index +1 ), see Figure B.5.(5);

(c. 3$)$ either $\alpha=2 \beta+1$ and $b^{2}+4 a(\beta+1)<0$, or $\alpha<2 \beta+1$ (or $\left.\Phi(x) \equiv 0\right)$, then the origin is either a focus, or a center, respectively (index+1).

Theorem. (Poincaré-Hopf Theorem, see (12]). Let $X$ be a vector field defined on a compact connected surface. If $X$ has finitely many critical points then the sum of the indices of all the critical points is independent of the vector field and equal to the Euler-Poincaré characteristic of the surface.

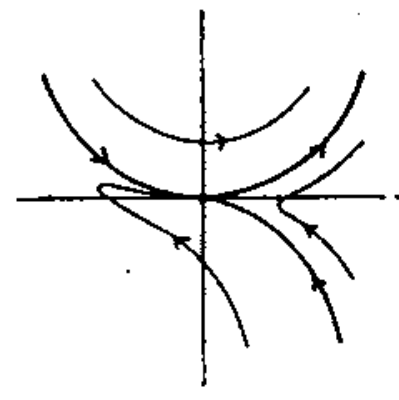

(1)

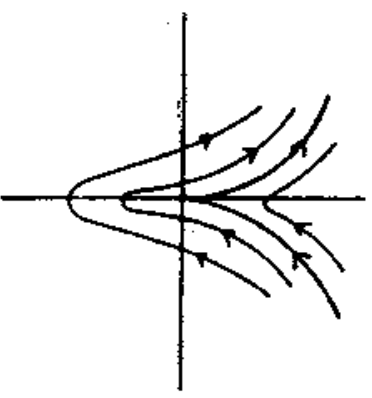

(2)

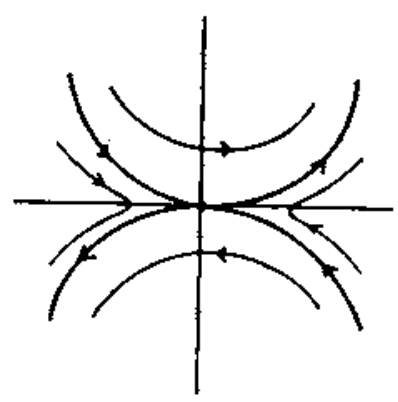

(3) 


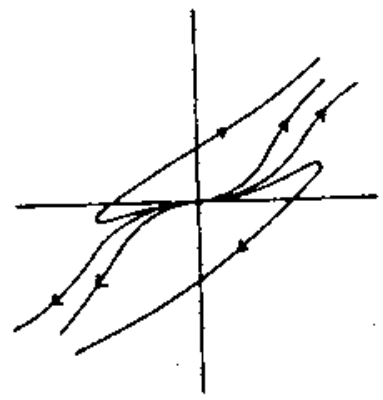

(4)

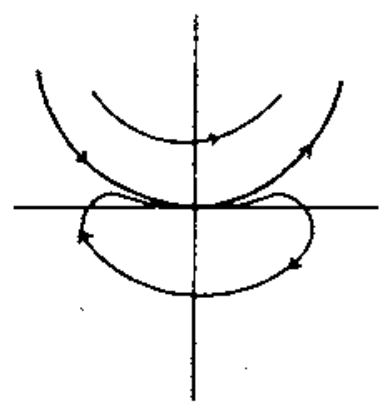

(5)

Figure B.5. The local behaviour near a singularity of type NE (we can reverse the orientation of the orbits).

\section{References}

1. A.F. ANDREEV, Investigation on the behaviour of the integral curves of a system of two differential equations in the neighbourhood of a singular point, Translation of A.M.S. 8 (1958), 183-207.

2. A.A. ANDRONOV, E.A. LEONTOVICH, I.I. GORDON AND A.L. MAIER, Qualitative theory of second-order dynamic systems, John Wiley \& Sons (1973), New York.

3. M.I.T. Camacho and C.F.B. Palmeira, Nonsingular quadratic differential equations in the plane, Transactions of A.M.S. 301 (1987), 845-859.

4. M.I.T. Camacho and C.F.B. Palmeira, Polynomial foliations of degree 3, Pitman Research Notes in Math. Series 160 (1987), 27-58, Longman.

5. A. Cima AND J. Llibre, Sistemas cúbicos homogéneos en el plano, Actas del VII Congreso de Ecuaciones diferenciales y Aplicaciones (1985), 97-103, Granada.

6. B. COli, A. Gasull AND J. Llibre, Some theorems on the existence, uniqueness and nonexistence of limit cycles for quadratic systems, J. Diff. Equations 67 (1987), 372-399.

7. A. Gasull, Sheng Li-Ren aND J. Llibre, Chordal quadratic systems, Rocky Mountain J. of Math. 16 (1986), 751-782.

8. E.A.V. GONZALES, Generic properties of polynomial vector fields at infinity, Trans. of Amer. Math. Soc 143 (1969), 201-222. 
9. A. HAEFLIGER AND G. REEB, Variétés (non separées) à une dimension et structures feuilletées du plan, Ens. Math. 3 (1957), 107-125.

10. W. Kaplan, Regular curve-families filling the plane, I, Duke Math. J. 7 (1940), 154-185.

11. W. KaPLAN, Regular curve-families filling the plane, II, Duke Math. J. $8(1941), 11-46$.

12. S. LeFSCHETZ, Differential equations: Geometric Theory, Interscience (1962), New York.

13. M.P. MULLER, Quelques propiétés des feuilletages polynomiaux du plan, Bol. Soc. Mat. Mex. 21 (1976), 6-14.

14. F. Plunivage, Espaces des feuilles de certaines structures fetrillet $\Theta e s$ planes, Colloq. Math. 18 (1967), 90-101.

15. S. SCheCter AND M. Singer, Planar polynomial foliations, Proc. of A.M.S. $79(1980), 649-656$.

16. I. SOTOMAYOR, Curvas definidas por equaçoes diferenciais no plano, Instituto de Matemática Pura e Aplicada (1981), Rio de Janeiro.

M. Carbonell: Dept. de Matemàtiques i Informàtica

Facultat de Ciències

Universitat de les Illes Balears

07071 Palma de Mallorca

SPAIN

J. Llibre: Dept de Matemàtiques

Facultat de Ciencies

Universitat Autonoma de Barcelona

08193 Bellaterra, Barcelona

SPAIN

Rebut el 17 de Gener de 1989 